\title{
Physical structure of the envelopes of intermediate-mass protostars
}

\author{
N. Crimier ${ }^{1}$, C. Ceccarelli ${ }^{1}$, T. Alonso-Albi ${ }^{2}$, A. Fuente ${ }^{2}$, P. Caselli ${ }^{3}$, D. Johnstone ${ }^{4,5}$, C. Kahane $^{1}$, B. Lefloch ${ }^{1}$,

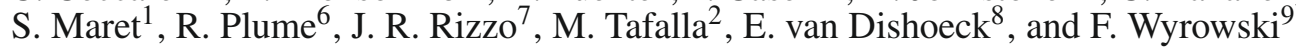 \\ 1 Laboratoire d'Astrophysique de l'Observatoire de Grenoble, BP 53, 38041 Grenoble Cedex 9, France \\ e-mail: ncrimier@obs.ujf-grenoble.fr \\ 2 Observatorio Astronómico Nacional (OAN, IGN), Apdo 112, 28803 Alcalá de Henares, Spain \\ 3 School of Physics \& Astronomy, E.C. Stoner Building, The University of Leeds, Leeds LS2 9JT, UK \\ ${ }^{4}$ National Research Council of Canada, Herzberg Institute of Astrophysics, 5071, West Saanich Road, Victoria, BC, V9E 2E7, \\ Canada \\ 5 Department of Physics \& Astronomy, University of Victoria, Victoria, BC, V8P 1A1, Canada \\ ${ }^{6}$ Department of Physics and Astronomy, University of Calgary, Calgary, T2N 1N4, AB, Canada \\ 7 Centro de Astrobiología (CSIC/INTA), Laboratory of Molecular Astrophysics, Ctra. Ajalvir km. 4, 28850 Torrejón de Ardoz, Spain \\ Leiden Observatory, PO Box 9513, 2300 RA Leiden, The Netherlands \\ 9 Max-Planck-Institut fur Radioastronomie, Auf dem Hügel 69, 53121 Bonn, Germany
}

Received 19 October 2009 / Accepted 27 March 2010

\section{ABSTRACT}

\begin{abstract}
Context. Intermediate mass protostars provide a bridge between low- and high-mass protostars. Furthermore, they are an important component of the UV interstellar radiation field. Despite their relevance, little is known about their formation process.

Aims. We present a systematic study of the physical structure of five intermediate mass, candidate Class 0 protostars. Our two goals are to shed light on the first phase of intermediate mass star formation and to compare these protostars with low- and high-mass sources.

Methods. We derived the dust and gas temperature and density profiles of the sample. We analysed all existing continuum data on each source and modelled the resulting SED with the 1D radiative transfer code DUSTY. The gas temperature was then predicted by means of a modified version of the code CHT96.

Results. We found that the density profiles of five out of six studied intermediate mass envelopes are consistent with the predictions of the "inside-out" collapse theory. We compared several physical parameters, like the power law index of the density profile, the size, the mass, the average density, the density at $1000 \mathrm{AU}$ and the density at $10 \mathrm{~K}$ of the envelopes of low-, intermediate, and high-mass protostars. When considering these various physical parameters, the transition between the three groups appears smooth, suggesting that the formation processes and triggers do not substantially differ.
\end{abstract}

Key words. stars: formation

\section{Introduction}

Stars are usually catalogued in three groups according to their mass: low-mass $\left(\lesssim 2 M_{\odot}\right)$, intermediate mass (IM) and high-mass $\left(\gtrsim 8 M_{\odot}\right)$. The high-mass stars are usually found to form in tight clusters (e.g. Hillenbrand \& Hartmann 1998), including many low-mass stars as well. Low-mass stars on their own can be formed in isolation or in loose groups of a few objects per cubic parsec (Gomez et al. 1993). While there is reasonable consensus on the overall process leading to the formation of isolated, low-mass stars (see e.g. Shu \& Adams 1987), the situation for high-mass star formation is much more confusing. The major problem is that a central object with $M \gtrsim 8 M_{\odot}$ produces a radiative pressure stronger than the gravitational pressure, which should stop the accretion. Therefore stars with a mass greater than $\sim 8 M_{\odot}$ cannot be formed in a simple-minded fully gravitationally driven process and other mechanisms need to be considered. Studying the differences in observed environments, several authors have proposed that high-mass stars are formed by the coalescence of lower-mass stars, while other authors favor "monolithic" formation (see for example the recent review by
Beuther et al. 2007), where turbulence or winds/outflows can play a major role (McKee \& Tan 2003; Krumholz et al. 2005).

The differences between low- and high-mass protostars are not only restricted to the dynamics of the formation mechanism. Notable differences also exist when it comes to the chemistry. Bottinelli (2006) compared the abundances of O-bearing complex organic molecules (COMs) present in Hot Cores surrounding high-mass protostars and in Hot Corinos around lowmass protostars. They concluded that the abundances of the O-bearing COMs, compared to methanol or formaldehyde, are about one order of magnitude higher in Hot Corinos than Hot Cores. In addition, significant differences in the abundances of molecules constituting the grain mantles $\left(\mathrm{H}_{2} \mathrm{O}, \mathrm{CO}\right.$, $\mathrm{CO}_{2}, \mathrm{CH}_{3} \mathrm{OH}, \mathrm{OCN}^{-}$) were observed between low- and highmass protostars (Boogert et al. 2004). Finally, in contrast to high-mass star forming regions, low-mass protostars present an extremely enhanced molecular deuteration, the so-called SuperDeuteration (e.g. Ceccarelli et al. 2007), not observed in highmass star forming regions.

The IM stars, namely stars whose mass lies in the 2 to $8 M_{\odot}$ range, are crucial in studies of star formation because they 
Table 1. The coordinates, the distances and the bolometric luminosities of the sources of the sample.

\begin{tabular}{llllll}
\hline \hline Source & CB3 & Cep E & IC1396 N & NGC7129 & Serpens \\
& mm & mm & BIMA 2 & FIRS 2 & FIRS 1 \\
\hline RA $(2000)$ & $00: 28: 42.1$ & $23: 03: 12.7$ & $21: 40: 41.8$ & $21: 43: 01.5$ & $18: 29: 49.8$ \\
Dec $(2000)$ & $56: 41: 59.4$ & $61: 42: 27.4$ & $58: 16: 13.5$ & $66: 03: 25.0$ & $01: 15: 18.4$ \\
Distance $(\mathrm{pc})$ & 2500 & 730 & 750 & 1250 & 230 \\
Luminosity $\left(L_{\odot}\right)$ & 1000 & 100 & 150 & 500 & 33 \\
\hline
\end{tabular}

provide the link between low- and high-mass stars (Di Francesco et al. 1997; Mannings \& Sargent 1997, 2000) and therefore can help to determine whether different formation processes are required. IM stars are also found in clusters (e.g. Testi \& Sargent 1998; Neri et al. 2007; Fuente et al. 2007), with a smooth transition towards the low-mass star, loose cluster regime for star masses around $3.5 M_{\odot}$ (Testi et al. 1999). These authors also conclude that IM stars mark the transition from the low-density aggregates of $\lesssim 10$ stars per cubic parsec of $\mathrm{T}$ Tauri stars to the dense clusters of $\gtrsim 10^{3}$ stars per cubic parsec associated with massive stars. Finally, IM stars are among the most dominant sources for the interstellar FUV field (e.g. Habing 1968; Gondhalekar \& Wilson 1975), which regulates the phases of the ISM in the Galaxy and, in turn, the overall Galaxy star formation process and history. In this context, the study of the formation of the IM stars is not only interesting in itself, but it can also greatly help the debate on the formation of high-mass stars. Indeed, due to their intermediate position, the study of IM protostars provides crucial information about the transition between the two formation regimes, as well as on the limits of the low-mass and high-mass formation scenarios.

Despite their importance, very little is known about the formation and first evolutionary stages of IM stars. Although some individual IM protostars have been studied in detail (Fuente et al. 2007; Neri et al. 2007; Hogerheijde et al. 1999; Schreyer et al. 2002), the situation is so bad that to date we neither have a satisfying sample of Class 0 IM objects, namely objects representing the first phases of stellar formation where the protostar is embedded in its envelope and its luminosity is dominated by the accretion luminosity, nor a systematic study of their physical structure, as in the case for low-mass and high-mass protostars. On the contrary, several previous studies have considered samples of low- and high-mass protostars. Relevant similar studies to the present IM one are those reported by Jørgensen et al. (2002) and Shirley et al. (2002), who targeted 7 and 11 low-mass Class 0 protostars respectively, and the systematic studies by Mueller et al. (2002), Hatchell \& van der Tak (2003), Williams et al. (2005) and Van der Tak et al. (2000), who targeted 31, 10, 32, and 9 high-mass protostars respectively. These authors derived the physical structure of the envelope for each protostar using continuum observations coupled with a radiative transfer code assuming a 1D spherical model. Combined, these studies cover a luminosity range of $1<L_{\odot}<22$, and $1 \times 10^{3}<L_{\odot}<2 \times 10^{6}$ for low- and high-mass protostars respectively, leaving a significant gap in luminosity and knowledge.

This paper aims to fill this gap, providing a systematic study of the physical structure of the envelopes of a sample of five IM protostars. In addition, this study will provide a valuable input for future work on the chemical structure of IM protostars (e.g. Alonso-Albi et al. 2010). The present work follows the method presented in Crimier et al. (2009) to study the IM protostars OMC2-FIR4. Namely, the combination of sub-millimeter to infrared observations with radiative transfer codes are used to derive the physical structure of each envelope. We selected a sample of five representative candidate Class 0 IM protostars: CB3-mm, Cep E-mm, IC1396 N BIMA 2, NGC7129 FIRS 2, and Serpens FIRS 1. These sources were selected using the following criteria:

- the luminosity is in the 30 to $1000 L_{\odot}$ range (corresponding to a $\sim 3$ to $9 M_{\odot}$ of ZAMS stars);

- the continuum maps at submillimetre and/or millimetre wavelengths are available;

- the source is closer than $2.5 \mathrm{kpc}$ to the Sun and isolated, namely no source of similar brightness in the submillimetre within an angular distance of $\sim 100^{\prime \prime}$.

The coordinates, the distances and the bolometric luminosities of the selected sources are listed in Table 1.

This article is organised as follows. Section 2 describes the observations used and the method and tools employed to derive the dust and gas density and temperature profiles. Section 3 first summarises the general results obtained toward the IM protostar sample. Next, for each source, background information, data available, and the results obtained are presented. In Sect. 4, the physical structure of the envelopes of IM protostars obtained in this study are compared with those obtained in previous studies of low- and high-mass protostar envelopes. In this section we also discuss the limitations of our modeling method. Finally, Sect. 5 concludes the article.

\section{General method}

In this section we present the general method used to derive the dust density and temperature profiles by modelling the continuum maps of each region, plus the Spectral Energy Distribution (SED) from the millimetre to the Mid-Infrared (MIR). We first describe the observations used in the analysis and how the brightness profiles and the SED are derived (Sect. 2.1), and then we present the modelling (Sect. 2.2).

\subsection{Continuum emission: observational data}

In our analysis we used the maps of the continuum emission at 850 and $450 \mu \mathrm{m}$ obtained at the $15 \mathrm{~m}$ James Clerk Maxwell Telescope (JCMT). In addition, we took into account the SED of each source from $24 \mu \mathrm{m}$ to $1300 \mu \mathrm{m}$ data obtained with the Spitzer, IRAS, JCMT and IRAM telescopes. Note that specific details (datasets, pipeline, uncertainties, contamination ... etc.) are given separately for each source in Sect. 3.

\section{- a) Continuum brightness profiles}

For each source we retrieved from the JCMT Science Archive $^{1}$ the $450 \mu \mathrm{m}$ and $850 \mu \mathrm{m}$ maps obtained by the focal-plane instrument SCUBA (Submillimetre CommonUser Bolometer Array). The data were reduced with the JCMT pipelines SURF V1.6-13, and KAPPA 1.9-7. When

1 http://www.jach.hawaii .edu/JCMT/archive/ 
N. Crimier et al.: The dust physical structure of an Intermediate mass protostars sample

Table 2. Continuum fluxes integrated over the envelope of each source and used in the SED analysis.

\begin{tabular}{llllll}
\hline \hline \multirow{2}{*}{ Source } & CB3 & Cep E & IC1396 N & NGC7129 & Serpens \\
& mm & mm & BIMA 2 & FIRS 2 & FIRS 1 \\
\hline$F_{24 \mu \mathrm{m}}(\mathrm{Jy})-$ Spitzer & $1.4 \pm 0.8$ & $6.0 \pm 3.0$ & $2.3 \pm 2.0$ & $6.0 \pm 3.0$ & $3.0 \pm 1.0$ \\
$F_{60 \mu \mathrm{m}}(\mathrm{Jy})-$ IRAS & $31 \pm 15$ & $61 \pm 30$ & - & - & - \\
$F_{70 \mu \mathrm{m}}(\mathrm{Jy})-$ Spitzer & $58 \pm 20$ & $100 \pm 40$ & - & $110 \pm 60$ & $135 \pm 50$ \\
$F_{100 \mu \mathrm{m}}(\mathrm{Jy})-$ IRAS & $110 \pm 55$ & $110 \pm 55$ & - & - & - \\
$F_{450 \mu \mathrm{m}}(\mathrm{Jy})-$ JCMT & $30 \pm 10$ & $44 \pm 10$ & $100 \pm 30$ & $80 \pm 40$ & $130 \pm 40$ \\
$F_{850 \mu \mathrm{m}}(\mathrm{Jy})-$ JCMT & $2.0 \pm 1.0$ & $4.1 \pm 1.3$ & $17 \pm 7.0$ & $4.8 \pm 2.0$ & $14 \pm 4.0$ \\
$F_{1300 \mu \mathrm{m}}(\mathrm{Jy})-$ IRAM30 m & - & $1.0 \pm 0.2$ & - & $2.2 \pm 0.5$ & - \\
\hline
\end{tabular}

multiple observations were available in the archive, we used the most sensitive ones. The angular resolution of the maps is approximately $7.5^{\prime \prime}$ and $14.8^{\prime \prime}$ at 450 and $850 \mu \mathrm{m}$ respectively (see Sect. 2.2 for more details). Based on the many previous JCMT published observations, the calibration uncertainty and noise levels are $\lesssim 10 \%$ and $0.04 \mathrm{Jy} \mathrm{beam}^{-1}$ at $850 \mu \mathrm{m}$ and $\lesssim 30 \%$ and $0.3 \mathrm{Jy}$ beam $^{-1}$ at $450 \mu \mathrm{m}$, respectively. To evaluate the continuum brightness profile (the brightness as a function of the distance from the envelope centre), we averaged the observed flux over annuli with the same distance from the centre of the source. The final uncertainties were evaluated taking into account the calibration uncertainty, the noise levels of the used maps, and the nonsphericity of the source. The non-sphericity of the source is taken into account via the computation of the standard deviation relative to the azimuthal average for each averaged flux of the brightness profile.

- b) SED

The SED points at $850 \mu \mathrm{m}$ and $450 \mu \mathrm{m}$ were obtained integrating the continuum flux profiles previously derived over the full envelope. We also considered the integrated flux at $24 \mu \mathrm{m}$ and $70 \mu \mathrm{m}$ extracted from the Spitzer Space Telescope Multiband Imaging Photometer (MIPS) maps. To this end, we retrieved the observations from the Spitzer Science archive ${ }^{2}$. The data reduction was performed using the pipeline S16.1.0. The evaluation of the fluxes was done with the same method employed for the maps at $850 \mu \mathrm{m}$ and $450 \mu \mathrm{m}$, namely deriving the brightness profiles from the maps and integrating over each annuli. We evaluated the uncertainties according to the instrument uncertainties and varying the outer radius of the envelope to account for the uncertainty in the envelope size. To do this we first set the outer radius equal to where the flux profile becomes flat (because dominated by the noise or the flux of the parent cloud) and varied this value up to $100 \%$. In general, the uncertainties remain relatively constant for variations larger than 30-50\%. Note that we checked a posteriori that our adopted radius and variation range is constitent with the outer radius obtained after the modelling. Finally, we also considered the IRAS fluxes at $60 \mu \mathrm{m}$ and $100 \mu \mathrm{m}$, respectively, taken from the literature or the IRAS Point Source Catalog v2.0 ${ }^{3}$. The SED fluxes of each source and their uncertainties are reported in Table 2.

\subsection{Continuum emission: modelling}

To derive the dust temperature and density profiles of the envelope, we used the $1 \mathrm{D}$ radiative transfer code DUSTY

\footnotetext{
2 http://ssc.spitzer.caltech.edu/archanaly/

${ }^{3}$ http://irsa.ipac. caltech.edu/cgi-bin/Gator/nph-dd? catalog=iraspsc
}

(Ivezic \& Elitzur 1997). Briefly, giving as input the temperature and size of the central object and a dust density profile, DUSTY self-consistently computes the dust temperature profile and the dust emission. The comparison between the computed $450 \mu \mathrm{m}$, $850 \mu \mathrm{m}$ and $1300 \mu \mathrm{m}$ brightness profiles (namely the brightness versus the distance from the centre of the envelope) and integrated SED against the observed brightness profiles and integrated SED (see previous paragraph) allows one to constrain the density profile and, consequently, the temperature profile of the envelope.

To be compared against the observations, the theoretical emission must be convolved with the beam pattern of each telescope. Following the recommendations for the JCMT, the beam is assumed to be a combination of three Gaussian curves: at $850 \mu \mathrm{m}$ we used HPBWs of $14.5^{\prime \prime}, 60^{\prime \prime}$, and $120^{\prime \prime}$, with amplitudes of $0.976,0.022$, and 0.002 respectively; at $450 \mu \mathrm{m}$ the $H P B W \mathrm{~s}$ are $8^{\prime \prime}, 30^{\prime \prime}$, and $120^{\prime \prime}$. with amplitude ratios of 0.934 , 0.06, and 0.006, respectively (Sandell \& Weintraub 2001).

In all sources we assumed that the envelope density follows a single index power law

$n(r)=n_{0} \times\left(\frac{r_{0}}{r}\right)^{\alpha}$,

where the power law index, $\alpha$, and the density, $n_{0}$, at $r_{0}$ are free parameters of the model. The envelope is assumed to start at a radius $r_{\text {in }}$ and extends to $r_{\text {out }}$. Both $r_{\text {in }}$ and $r_{\text {out }}$ are additional free parameters of the model. The last input into DUSTY is the temperature of the central source, $T_{*}$, here assumed to be $5000 \mathrm{~K}$. We verified that the choice of this last parameter does not influence the results. Indeed, given the high optical thickness of the envelopes at the wavelengths where the central source emits, the model outputs are not sensitive to the $T_{*}$ value. Finally, the opacity of the dust as function of the wavelength is another parameter of DUSTY. Following numerous previous studies (van der Tak et al. 1999; Evans et al. 2001; Shirley et al. 2002; Young et al. 2003), we adopted the dust opacity calculated by Ossenkopf \& Henning (1994), specifically their OH5 dust model, which refers to grains coated by ice. We also obtained a lower limit to $T_{\text {in }}$ (the temperature at $R_{\text {in }}$ ) of $300 \mathrm{~K}$ : any higher value for $T_{\text {in }}$ would give similar results. Again, given the high optical thickness of the envelope at short wavelengths and the low contribution from the dust at $T>300 \mathrm{~K}$ to the sub-millimetre emission (as the NIR emission is mainly from the very inner part, i.e. a small volume), increasing $T_{\text {in }}$ does not make a difference to the resulting best-fit.

In summary, the output of DUSTY depends on four free parameters: $\alpha, n_{0}, r_{\text {in }}$ and $r_{\text {out }}$. In practice, the DUSTY input parameters are the power law index, $\alpha$, the optical thickness at $100 \mu \mathrm{m}$, $\tau_{100}$, the ratio between the inner and outer radius, $Y\left(=r_{\text {out }} / r_{\text {in }}\right)$ and the temperature at the inner radius $T_{\text {in }}$. The optical thickness is, in turn, proportional to the dust column density which depends on $n_{0}$ and the physical thickness of the envelope. Note that 
Table 3. Range of the input parameters to DUSTY covered in the present study.

\begin{tabular}{lll}
\hline \hline Parameter & Range & Step \\
\hline$\alpha$ & $0.2-2.5$ & 0.1 \\
$Y$ & $50-2000$ & 10 \\
$\tau_{100}$ & $0.1-10$. & 0.1 \\
$T_{\text {in }}$ & $300 \mathrm{~K}$ & \\
$T_{*}$ & $5000 \mathrm{~K}$ & \\
\hline
\end{tabular}

Notes. The range of the $\alpha, Y$ and $\tau_{100}$ parameters are covered by increasing by a constant value at each step of the grid reported in the third column. Note that $T_{\text {in }}$ and $T_{*}$ remain fixed as they do not influence the results (see text).

because the beam sizes of the available maps are relatively large ( $\geq 7.5^{\prime \prime}$, which corresponds to a radius of $\geq 3250$ AU for a source at a distance of $1000 \mathrm{pc}$ ), the inner regions of the envelopes are relatively unconstrained by the available observational data. Finally, as explained in Ivezic \& Elitzur (1997), DUSTY gives scaleless results (which make it very powerful because the same grid of models can be applied to many different sources). To compare the DUSTY output with actual observations it is necessary to scale the output by the source bolometric luminosity, $L_{\text {bol }}$, and the distance to the source. Note that the bolometric luminosity is estimated by integrating the emission across the full spectrum. By definition, this can only be done when the entire SED is known. This is exactly one of the outputs of the modelling. Therefore, we re-evaluate the luminosity of each source iteratively from the best-fit model, by minimising the $\chi^{2}$.

We ran a grid of models to cover the parameter space as reported in Table 3.

The best-fit model is found by minimising the $\chi^{2}$ with an iterated two-steps procedure. First, we used the observed brightness profiles at $450 \mu \mathrm{m}$ and $850 \mu \mathrm{m}$ to constrain $\alpha$ and $Y$, assuming a value for $\tau_{100}$. The $\chi_{\text {red }}^{2}$ computed during this first step are reported as $\chi_{\text {red }}^{2}$-maps in Table 4 . Second, we constrain the optical thickness $\tau_{100}$ by comparing the computed and observed SED, assuming the $\alpha$ and $Y$ of the previous step. The new $\tau_{100}$ is used for the next iteration and the process is repeared. The $\chi_{\text {red }}^{2}$ computed during this second step are reported as $\chi_{\text {red }}^{2}$-SED in Table 4. In practice, the iteration converges in two steps. This occurs because the normalised brightness profiles are only very weakly dependent on $\tau_{100}$, while they are very dependent on the sizes of the envelope and on the slope of the density profile (see also Jørgensen et al. 2002; Schöier et al. 2002; Crimier et al. 2009). On the contrary, the optical thickness depends mostly on the absolute column density of the envelope, which is constrained by the SED.

\subsection{Gas temperature profile: model description}

Ceccarelli et al. (1996), Crimier et al. (2009) and Doty \& Neufeld (1997) showed that the gas can be thermally decoupled from the dust in the inner regions of low-, intermediate and high-mass protostellar envelopes. The decoupling occurs mainly in the inner part of the envelopes: for example whereas $T_{\text {dust }} \sim 150 \mathrm{~K}$ at $200 \mathrm{AU}, T_{\text {gas }} \sim 90 \mathrm{~K}$. Note that in the outer envelope, where $T_{\text {dust }} \lesssim 100 \mathrm{~K}$, gas and dust temperature only differ by a few percents (see discussion in Crimier et al. 2009). The reason for this decoupling is the high water abundance in the gas phase caused by the sublimation of grain mantles. We therefore explicitly computed the gas temperature profile of the envelope surrounding each source by finding the equilibrium temperature obtained by equating the gas cooling and heating terms at each radius. Following the method described by Ceccarelli et al. (1996), we considered heating from the gas compression (due to the collapse), dust-gas collisions, photo-pumping of $\mathrm{H}_{2} \mathrm{O}$ and $\mathrm{CO}$ molecules by the IR photons emitted by the warm dust close to the centre, and cosmic rays ionisation which is a minor heating term in protostellar envelopes. The cooling is mainly due to the rotational lines from $\mathrm{H}_{2} \mathrm{O}$ and $\mathrm{CO}$, plus the fine structure lines from $\mathrm{O}$. The gas temperature therefore depends on the abundance of these three species. In practice, only the water abundance is an important parameter of the model, because the $\mathrm{CO}$ and $\mathrm{O}$ lines are optically thick and LTE populated in the range of $\mathrm{CO}$ and $\mathrm{O}$ abundances typical of protostellar envelopes, while the water levels are sub-thermally populated (non-LTE). In the non-LTE regime the water levels are excited by collisional processes and de-excited by radiation while in LTE regime the levels are mainly excited and de-excited by collisions. Therefore, a photon emitted in LTE regime in an optically thick region will be absorbed and then the absorber will be de-excited by collisions while in non-LTE regime the absorbed photon will possibly be re-emitted. In this case we say that the water lines are effectively optically thin. For this reason we computed two cases for the water abundance, as it is generally poorly constrained in protostellar envelopes and totally unconstrained in intermediate mass protostars. We adopted a step function for the water abundance profile to simulate the jump caused by ice sublimation. The jump is assumed to occur at $100 \mathrm{~K}$. We considered the $\mathrm{H}_{2} \mathrm{O}$ abundance (with respect to $\left.\mathrm{H}_{2}\right) X\left(\mathrm{H}_{2} \mathrm{O}\right)_{\text {in }}$ in the inner envelope, where $T \geq 100 \mathrm{~K}$, equal to $10^{-5}$ and $10^{-6}$, fixing the water abundance in the outer region, $X\left(\mathrm{H}_{2} \mathrm{O}\right)_{\text {out }}$, at $10^{-7}$. The $\mathrm{CO}$ and $\mathrm{O}$ abundances were fixed at the standard values found in molecular clouds, i.e. $1 \times 10^{-4}$ (Frerking et al. 1982) and $5 \times 10^{-4}$ (Caux et al. 1999; Vastel et al. 2000), respectively. Note that because the $\mathrm{O}$ and $\mathrm{CO}$ lines are mostly optically thick the exact value of their abundance is not important. To compute the cooling from the lines we used the code described in Ceccarelli et al. (2003, 1996) and Parise et al. (2005). Briefly, the line cooling is computed with an escape probability method, which takes into account the dust level pumping and the line optical depths at each point of the envelope by integrating over the solid angle. A recent description of the code is reported in Crimier et al. (2009, 2010). The same code has been used in several past studies, whose results have been substantially confirmed by other groups (e.g. the analysis on IRAS16293-2422 by Schöier et al. 2002).

For the collisional coefficients of water with hydrogen molecules, we used the data by Faure et al. (2007) and Faure \& Josselin (2008) available for the temperature range 20-5000 K. Because the ortho-to-para conversion process of $\mathrm{H}_{2}$ is chemical rather than radiative, the Ortho-to-Para Ratio (OPR) $\mathrm{H}_{2}$, which the water population depends on, is highly uncertain. The recent analysis of $\mathrm{H}_{2} \mathrm{CO}$ observations towards a cold molecular cloud by Troscompt et al. (2009) confirms theoretical estimates (e.g. Flower et al. 2006) that in molecular clouds the $\mathrm{H}_{2}$ OPR is lower than 1. Lacking specific observations towards protostars, here we assume that $\mathrm{H}_{2}$ OPR is in Local Thermal Equilibrium and therefore follows the Boltzmann distribution

$$
O P R=\frac{\left(2 I_{\mathrm{o}}+1\right) \Sigma(2 J+1) \exp \left(-\frac{E_{\mathrm{o}}(J)}{k T}\right)}{\left(2 I_{\mathrm{p}}+1\right) \Sigma(2 J+1) \exp \left(-\frac{E_{\mathrm{p}}(J)}{k T}\right)},
$$

where $I_{\mathrm{o}}$ and $I_{\mathrm{p}}$ are the total nuclear spin, corresponding to whether the hydrogen nuclear spins are parallel $\left(I_{\mathrm{o}}=1, \uparrow \uparrow\right)$ or anti-parallel $\left(I_{\mathrm{p}}=0, \uparrow \downarrow\right)$. The sum in the numerator and 
N. Crimier et al.: The dust physical structure of an Intermediate mass protostars sample

Table 4. Summary of the dust radiative transfer analysis for the five IM protostars.

\begin{tabular}{|c|c|c|c|c|c|c|}
\hline \multicolumn{7}{|l|}{ Fixed input parameters } \\
\hline Stellar temperature, $T_{\star}$ & $5000 \mathrm{~K}$ & & & & & \\
\hline Dust temperature at $r_{\text {in }}, T_{\text {in }}$ & $300 \mathrm{~K}$ & & & & & \\
\hline Dust opacity (OH5) at $100 \mu \mathrm{m}, \kappa_{100}$ & $86.5 \mathrm{~cm}^{2} \mathrm{~g}^{-1}$ & & & & & \\
\hline \multicolumn{7}{|c|}{ Best-fit parameters \& physical quantities } \\
\hline Source & CB3 & Cep E & IC1396 N & NGC7129 & Serpens & OMC2 \\
\hline & $\mathrm{mm}$ & $\mathrm{mm}$ & BIMA2 & FIRS 2 & FIRS 1 & FIR4 \\
\hline Distance, $(\mathrm{pc})$ & 2500 & 730 & 750 & 1250 & 230 & 450 \\
\hline Luminosity, $\left(L_{\odot}\right)$ & 1000 & 100 & 150 & 500 & 33 & 1000 \\
\hline Dust optical depth at $100 \mu \mathrm{m}, \tau_{100}$ & 5.8 & 5.0 & 1.4 & 2.3 & 3.0 & 0.6 \\
\hline Density power law index, $\alpha$ & 2.2 & 1.9 & 1.2 & 1.4 & 1.5 & 0.6 \\
\hline Envelope thickness, $Y\left(=r_{\text {out }} / r_{\text {in }}\right)$ & 400 & 500 & 630 & 180 & 200 & 120 \\
\hline$\chi_{\text {red }}^{2}$-maps & 0.43 & 0.11 & 0.55 & 0.21 & 0.20 & 1.2 \\
\hline$\chi_{\mathrm{red}}^{2}-\mathrm{SED}$ & 0.85 & 0.80 & 1.2 & 0.87 & 4.4 & 0.55 \\
\hline Inner envelope radius, $r_{\text {in }},(\mathrm{AU})$ & 260 & 70 & 50 & 100 & 30 & 100 \\
\hline Outer envelope radius, $r_{\text {out }}$, (AU) & 103000 & 35800 & 29600 & 18600 & 5900 & 12000 \\
\hline Radius at $T_{\text {dust }}=100 \mathrm{~K}, r_{100 \mathrm{~K}},(\mathrm{AU})$ & 700 & 223 & 180 & 373 & 102 & 440 \\
\hline $\mathrm{H}_{2}$ density at $r_{100 \mathrm{~K}}, n_{0},\left(\mathrm{~cm}^{-3}\right)$ & $7.5 \times 10^{7}$ & $2.0 \times 10^{8}$ & $4.3 \times 10^{7}$ & $4.4 \times 10^{7}$ & $2.2 \times 10^{8}$ & $4.3 \times 10^{6}$ \\
\hline Envelope mass, $M_{\mathrm{env}},\left(M_{\odot}\right)$ & 120 & 35 & 90 & 50 & 5.0 & 30 \\
\hline$\left(T_{\text {dust }}-T_{\text {gas }}\right)_{\max },(\mathrm{K})$ & 6 & 5 & 40 & 40 & 10 & 150 \\
\hline
\end{tabular}

Notes. The top section lists the fixed input parameters, the lower section reports the best-fit parameters, and some relevant physical quantities corresponding to the best-fit model. The last column reports the same parameters for the IM protostar OMC2-FIR4, found with a similar analysis (Crimier et al. 2009), for comparison.

denominator extends over all ortho and para levels $J$, respectively. Similarly to $\mathrm{H}_{2}$, water comes in the ortho and para forms. In these cases, because the water is the dominant gas coolant only in the regions where the dust temperature exceeds $100 \mathrm{~K}$, we assumed an OPR equal to 3, strictly valid for gas temperatures higher than $60 \mathrm{~K}$. Because the water lines are optically thick, the cooling depends on the velocity field, assumed to be that of an envelope collapsing in free-fall towards a central object with a mass $M_{\star}$. Here, we assumed that the entire envelope is in infall. The masses $M_{\star}$ used for each source are reported in Table 4. They were derived analytically, following the equation in Stahler et al. (1986) which links the mass of the central object to the bolometric luminosity, the accretion rate, the mass and the radius of the envelope. Basically, the equation assumes that the luminosity is entirely due to the gravitational energy released in the collapse and uses the computation of the hydrostatic core radius of the protostar by Stahler et al. (1986). We checked the influence of our results against this assumption, running cases with $M_{\star}$ fixed at $2 M_{\odot}$. The difference in the gas temperature between the two cases never exceeds $1 \%$.

\subsection{Water line observations}

In order to constrain the water abundance, which is very important for computing the gas temperature, we considered observations obtained by the Long Wavelength Spectrometer (LWS) aboard the Infrared Space Observatory (ISO) in the $45 \mu \mathrm{m}$ to $200 \mu \mathrm{m}$ range, where several water lines emit. All sources excepted CB3 were observed with the LWS. In two sources, Cep E-mm and Serpens FIRS 1, several water lines were detected and their analysis has been reported by Moro-Martín et al. (2001) and Larsson et al. (2002), respectively. As discussed by these authors, given the relatively large beam of the LWS, the water line fluxes are due to the combination of many components along the line of sight: outflows, multiple sources and PhotoDissociation Regions. The measured $\mathrm{H}_{2} \mathrm{O}$ line fluxes are thus upper limits to the fluxes from the envelopes and we checked that our predictions do not exceed the observed fluxes. For two other sources, IC1396 N BIMA 2 and NGC7129 FIRS 2, we retrieved the LWS grating spectra (spectral resolution $\Delta v / v \sim 200$ ) from the ISO Data Archive 4 and extracted the upper limits to the flux of the brightest $\mathrm{H}_{2} \mathrm{O}$ lines. Table 5 summarises the water line ISO observations for each source.

\section{Results : Dust and gas density and temperature profiles}

The general results of our analysis are:

- The continuum brightness profiles and the SEDs at wavelengths larger than $60 \mu \mathrm{m}$ for the five IM-protostars of our sample can be reproduced by spherical, single index, power law density models. On the contrary, in all sample sources, this class of models fails to reproduce the $24 \mu \mathrm{m}$ flux, underestimating it by 1 to 3 orders of magnitude. The possible causes and implications of this failure are discussed in detail in Sect. 4. The $\chi_{\text {red }}^{2}$-maps and $\chi_{\text {red }}^{2}$-SED obtained for each source are reported in Table 4 . The $\chi_{\text {red }}^{2}$-maps ranges from 0.11 to 0.55 for the sources modelled in this paper. The $\chi_{\text {red }^{-}}^{2}$ SED ranges from 0.8 to 4.4 for the sources modeled in this paper. Note that because the flux at $24 \mu \mathrm{m}$ is underestimated by several orders of magnitude, the $\chi_{\text {red }}^{2}$-SED value is mainly driven by this point.

- The power law index for the five sample sources varies between 1.2 and 2.2, with an average value equal to 1.6.

- The envelope radius varies between 6000 AU for the lowest luminosity and closest source, Serpens FIRS 1, and $1 \times 10^{5} \mathrm{AU}$ for the brightest and farthest source, CB3-mm.

- The radius where $T_{\text {dust }}=100 \mathrm{~K}$ lies between 100 (Serpens FIRS 1) and 700 (CB3-mm) AU.

- The density at $T_{\text {dust }}=100 \mathrm{~K}$ varies, from 0.4 to $2 \times 10^{8} \mathrm{~cm}^{-3}$.

- The envelope mass ranges from $5 M_{\odot}$ (Serpens FIRS 1) to $120(\mathrm{CB} 3-\mathrm{mm}) M_{\odot}$.

${ }^{4}$ http://iso.esac.esa.int/ida/ 
Table 5. The brightest water lines predicted by the our model considering $X\left(\mathrm{H}_{2} \mathrm{O}\right)_{\text {in }}=1 \times 10^{-5}$ and $X\left(\mathrm{H}_{2} \mathrm{O}\right)_{\text {out }}=1 \times 10^{-7}$ compared with the ISO LWS observations. The upper limits refer to $3 \sigma$. All fluxes are in $10^{-12} \mathrm{erg} \mathrm{s}^{-1} \mathrm{~cm}^{-2}$ units.

\begin{tabular}{|c|c|c|c|c|c|c|c|c|c|c|c|}
\hline \multicolumn{2}{|l|}{ Source } & \multirow{2}{*}{$\frac{\mathrm{CB} 3}{\mathrm{~mm}}$} & & \multicolumn{2}{|l|}{ Cep E } & \multicolumn{2}{|l|}{ IC1396 N } & \multicolumn{2}{|l|}{ NGC7129 } & \multicolumn{2}{|l|}{ Serpens } \\
\hline & & & & $\mathrm{mm}$ & & BIMA 2 & & FIRS 2 & & FIRS 1 & \\
\hline $\begin{array}{c}\lambda \\
(\mu \mathrm{m})\end{array}$ & $\begin{array}{c}\text { Transition } \\
J_{K_{-} K_{+}}-J_{K_{-}^{\prime} K_{+}^{\prime}}^{\prime}\end{array}$ & Model & LWS & Model & LWS & Model & LWS & Model & LWS & Model & LWS \\
\hline ortho & & & & & & & & & & & \\
\hline 75.38 & $3_{21}-2_{12}$ & 0.3 & - & 0.2 & $1.4 \pm 0.3$ & 0.08 & $<3.1$ & 0.2 & $<1.7$ & 0.5 & $2 \pm 0.4$ \\
\hline 108.07 & $2_{21}-1_{10}$ & 0.1 & - & 0.1 & $1.8 \pm 0.5$ & 0.07 & $<2.3$ & 0.1 & $<1.5$ & 0.4 & $1.5 \pm 0.4$ \\
\hline 113.54 & $4_{14}-3_{03}$ & 0.1 & - & 0.1 & $2.2 \pm 0.5$ & 0.04 & $<1.2$ & 0.08 & $<1.0$ & 0.2 & $2.9 \pm 0.9$ \\
\hline 174.62 & $3_{03}-2_{12}$ & 0.06 & - & 0.07 & $1.6 \pm 1$ & 0.06 & $<2.6$ & 0.08 & $<0.8$ & 0.2 & $2 \pm 0.2$ \\
\hline $\begin{array}{l}179.53 \\
\text { para }\end{array}$ & $2_{12}-1_{01}$ & 0.09 & - & 0.1 & $2.9 \pm 0.3$ & 0.2 & $<2.2$ & 0.2 & $<1.1$ & 0.5 & $1.4 \pm 0.2$ \\
\hline 89.99 & $3_{22}-2_{11}$ & 0.2 & - & 0.1 & $1 \pm 0.2$ & 0.04 & $<2.4$ & 0.1 & $<1.5$ & 0.3 & $2.4 \pm 0.6$ \\
\hline 100.98 & $2_{20}-1_{11}$ & 0.1 & - & 0.1 & $<0.5$ & 0.04 & $<1.0$ & 0.09 & $<0.6$ & 0.3 & $2.6 \pm 0.5$ \\
\hline
\end{tabular}

- The mass of the central object is estimated to be between $0.1 M_{\odot}$ (Serpens FIRS 1) and 6 (CB3-mm) $M_{\odot}$.

- The gas and the dust are thermally coupled across the envelope in three of our sources, with temperature differences lower than 5 K. For IC1396 N BIMA 2 (Sect. A.3) and NGC7129 FIRS 2 (Sect. A.4) the difference can reache $40 \mathrm{~K}$.

- The predicted $\mathrm{H}_{2} \mathrm{O}$ lines are consistent with the ISO upper limits of Sect. 2.4.

Table 4 summarises the best-fit parameters and some relevant physical quantities derived from the dust radiative transfer analysis of each source, Table 5 lists the water line predictions. Note that given the relatively large beam, the ISO observations are contaminated by the emission from outflows, multiples sources, and Photo-Dissociation Regions and are therefore considered only as upper limits.

The appendix describes in detail the source background, the data included in the analysis, and the derived physical structure (gas and dust density and temperature profiles) for each source.

\section{Discussion}

\subsection{The link between low- and high-mass protostars}

One of the major goals of this work is to verify whether intermediate mass protostars provide a link between low- and high-mass star formation. In this section, we analyse whether the parameters describing the protostellar envelope structure (power law index, dust temperature at a given distance, envelope mass...) depend on the luminosity, and hence the mass, of the future central star.

Figure 1 plots key parameters of the envelope structure (the power law index of the density profile, $\alpha$, the total mass, $M_{\mathrm{env}}$, the outer radius, $r_{\text {out }}$, and the average density, $\langle n\rangle$ of the envelope) for low-, intermediate, and high-mass protostars as a function on the bolometric luminosity of each source. The ensemble of the plotted sources covers six orders of magnitude in luminosity, from about $1 L_{\odot}$ to $\sim 10^{6} L_{\odot}$. The plotted data are from the present study (Table 4) and Crimier et al. (2009) for the intermediate mass protostars, Jørgensen et al. (2002), Shirley et al. (2002) and Crimier et al. (2010) for the low-mass protostars, and Van der Tak et al. (2000), Hatchell \& van der Tak (2003), Williams et al. (2005) and Mueller et al. (2002) for the highmass protostars. Note that Jørgensen et al. (2002), Williams et al. (2005), Hatchell \& van der Tak (2003), and Crimier et al. (2009, 2010), as well as the present study, use the DUSTY code in the analysis. The correlation coefficients and probability for a chance correlation between the pair of parameters considered in Fig. 1 are reported in Table 6. The plots and quantities shown in Fig. 1 and Table 6, respectively, lead to the following remarks.

\section{Density power law index $\alpha$ :}

The density power law index $\alpha$ is similar for low, intermediate and high-mass protostars. In all three cases the average $\alpha$ is 1.5 . We find that about $60 \%$ of the protostars are well modelled by envelopes with $1.5 \lesssim \alpha \lesssim 2.0$. This indicates that $60 \%$ of the protostar envelopes are consistent with the standard model of free-fall collapse from an initially singular isothermal sphere, the so-called inside-out model (Shu 1977). However, it appears that $35 \%$ of the sample are reproduced by envelopes with much smaller indexes, namely $0.5 \lesssim \alpha \lesssim 1.5$. The theoretical interpretation of theses low $\alpha$ values is not straightforward. The phenomenon was already noted in previous studies of low-mass protostars (e.g. Andre et al. 1993; Chandler et al. 1998; Motte \& André 2001). Various hypotheses to explain theses low $\alpha$ values have been evoked in the literature. One possibility is that the envelopes with low $\alpha$ are described by the collapse of an initially logotropic sphere, rather than a singular isothermal sphere (Lizano \& Shu 1989; McLaughlin \& Pudritz 1996, 1997; Andre et al. 2000). Basically, the logotropic model assumes that the gas pressure across the initial condensation depends logarithmically on the density, giving rise to a flatter density profile in the static part and at the infall/static interface of the envelope. Because the structure of prestellar cores is well described by a flat density profile in the inner region $(\alpha \sim 0)$ and a power law index of $\sim 2$ in the outer part (Visser et al. 2002; Andre et al. 2000), it has been mentioned in the past that a lower value of $\alpha$ could probe a younger protostar. However, later systematic studies have not supported this interpretation (e.g. Jørgensen et al. 2002). Another possible explanation is that the envelope is flattened for example because of the presence of a magnetic field (e.g. Li \& Shu 1996; Hennebelle \& Fromang 2008). However, testing this hypothesis is not trivial as it requires to solve the radiative transfer in a $2 \mathrm{D}$ geometry. A simple toy model which assumes constant temperature and optically thin emission suggests that $\alpha$ cannot be lower than 1 even in the extreme case of a flattened structure with axis ratio of $1: 10$ seen face-on. The few cases with $\alpha \geq 2$ are easier to explain and may be due to the presence of one or more high-density structures, like discs (Jørgensen et al. 2007), embedded within the envelope.

\section{Envelope mass $M_{\mathrm{env}}$ :}

It is very clear from Fig. 1 that more luminous sources have larger envelope mass. Hatchell \& van der Tak (2003) report the 
N. Crimier et al.: The dust physical structure of an Intermediate mass protostars sample
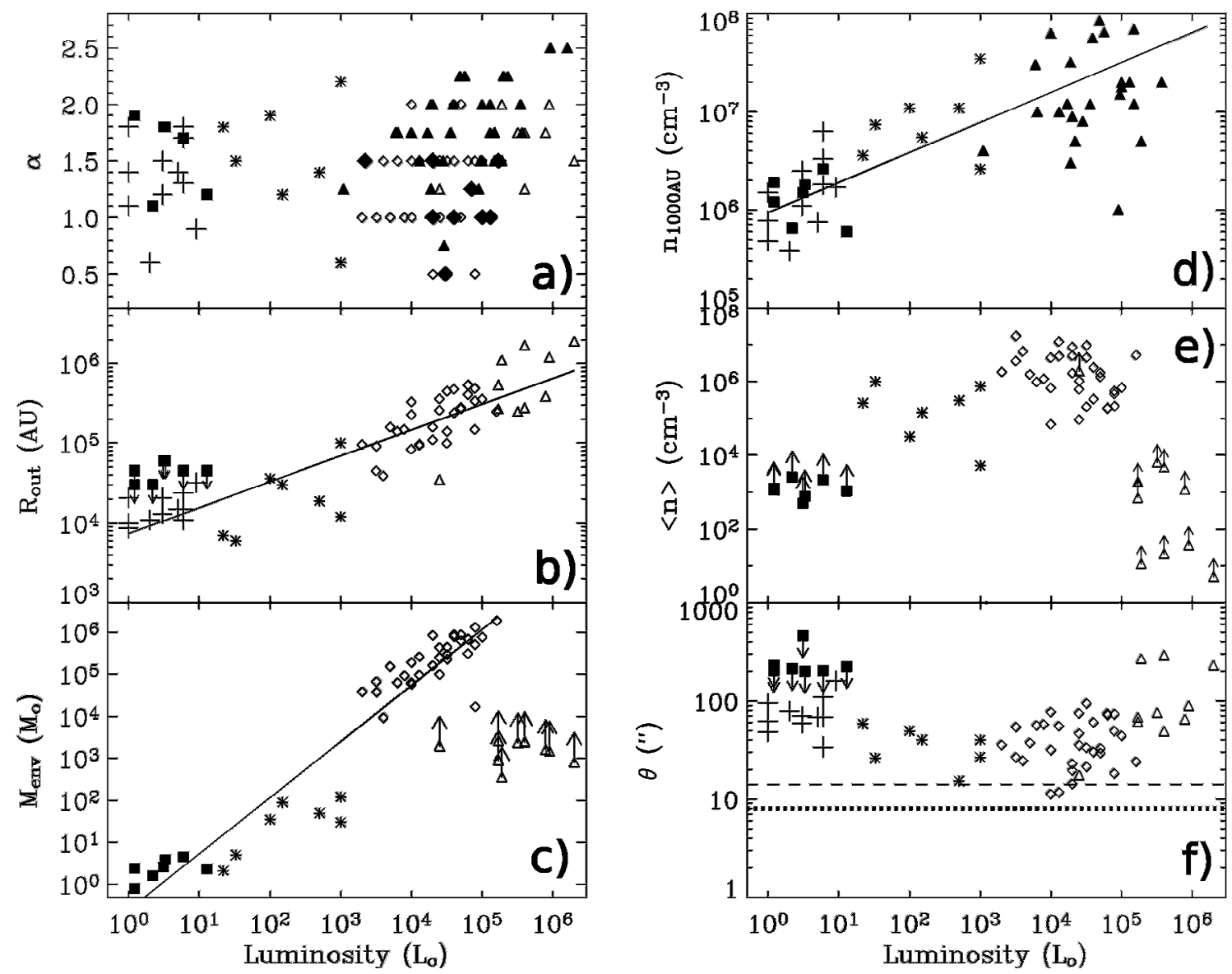

Fig. 1. Plot of physical quantities within the envelopes surrounding low-, intermediate, and high-mass protostars as a function of their bolometric luminosity. Each symbol represents a different group of sources as follows: crosses: Jørgensen et al. (2002); filled squares: Shirley et al. (2002); stars: this study and Crimier et al. (2009, 2010); diamonds: Williams et al. (2005); filled diamonds: Van der Tak et al. (2000); triangles: Hatchell \& van der Tak (2003); filled triangles: Mueller et al. (2002). Symbols with arrows represent upper/lower limits. The solid line represents the linear fit of the points obtained when the correlation is significant (Table 6). The dashed line and the dotted line on panel f) represent the angular resolution of the SCUBA maps at 850 and $450 \mu \mathrm{m}$, respectively, which were used in all the in the studies reported in this panel.

mass of the envelopes within 1 pc (empty triangles in Fig. 1). Therefore, the envelope masses from Hatchell \& van der Tak (2003) are considered as lower limits for $M_{\mathrm{env}}$, and are not taken into account in the correlation coefficient computation. The correlation coefficient is 0.96 with a probability for a chance correlation of $\sim 10^{-20}$, pointing out a strong relation between the two variables. Assuming that the luminosity is entirely due to the gravitational energy released, the luminosity-envelope mass relation suggests that a similar relation exists between the mass accretion rate and the mass of the envelope. However, while the result that more luminous sources have larger envelope mass agrees with theoretical expectations, it should be considered with caution. Indeed, the observed correlation between two quantities does not necessarily imply a physical correlation. The envelope-mass-derivation differs between studies. Furthermore, uncertainties in the distance to sources, particularly in the high-mass cases (uncertainties of several $\mathrm{kpc}$ ), and resolution limits of distant objects can introduce errors and significant observational bias.

Envelope radius $r_{\text {out }}$ :

Similar to $M_{\mathrm{env}}$, the outer radius of the envelope $r_{\text {out }}$ increases with increasing luminosity, varying from $\sim 10^{4} \mathrm{AU}$ for low-mass protostars to $\sim 10^{6} \mathrm{AU}$ for high-mass protostars. The correlation coefficient between $r_{\text {out }}$ and the luminosity is very high, 0.90, with a probability for a chance correlation of $10^{-22}$. Note that we checked that the relation is real and not simply due to the distance to the source combined to the angular resolution limits. This point is illustrated by the plot of the measured angular size, $\theta$, of the sources as function of the luminosity in Fig. 1 (panel f). The figure shows that $\theta$ is not decreasing with luminosity, which excludes the possibility of an observational bias due to the limited angular resolution of the observations.

Average density $\langle n\rangle$ :

The average density $\langle n\rangle$ in the envelope is derived from $r_{\text {out }}$ and $M_{\text {env }}$ for each source. Unfortunately, $\langle n\rangle$ could be derived only for intermediate and some high-mass sources, giving an average of about $4 \times 10^{5}$ and $3 \times 10^{6} \mathrm{~cm}^{-3}$, respectively, over a spread in luminosity of about four orders of magnitude. Although the average $\langle n\rangle$ is one order of magnitude higher in high-mass protostars versus intermediate mass protostars, the correlation coefficient between $\langle n\rangle$ and the luminosity is only 0.55 . The lower limits for low-mass and the highest mass protostars do not allow any firm conclusion, but it seems that there is little difference in the average density of these envelopes.

\section{Density at $1000 \mathrm{AU}$ :}

There is an apparent increase of the density at $1000 \mathrm{AU}$ with increasing luminosity, going from about $\sim 10^{6}$ to $\sim 10^{8} \mathrm{~cm}^{-3}$ 
A\&A 516, A102 (2010)

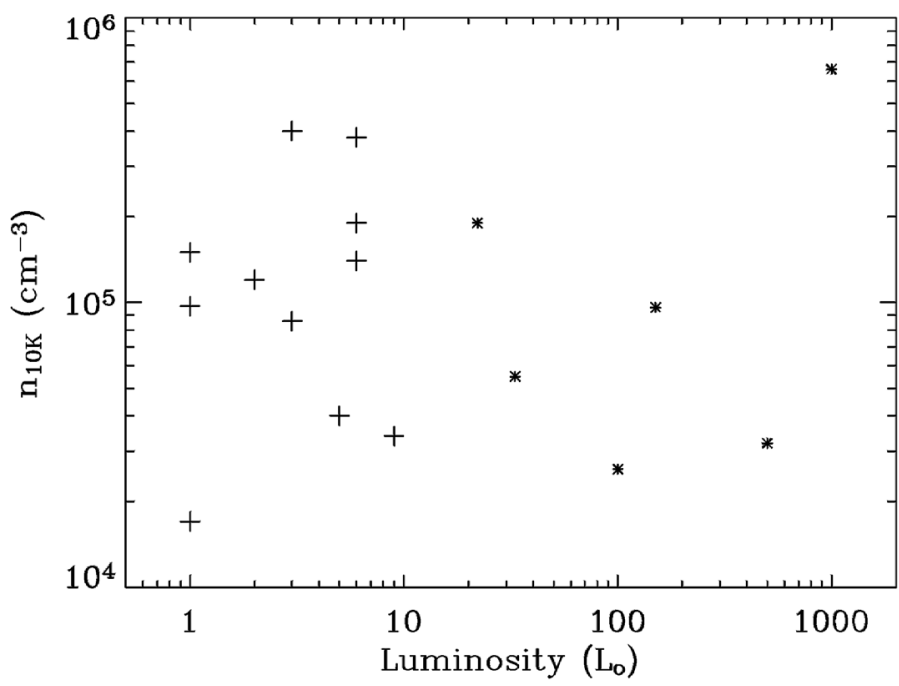

Fig. 2. Plot of the density at $10 \mathrm{~K}$ as function of the bolometric luminosity of a sample of low- and intermediate mass protostars. Data are from Jørgensen et al. (2002), (crosses), and the present study, (stars).

for source luminosity varying from 1 to $\sim 10^{5} L_{\odot}$. There are, however, some exceptions on the high-mass side. Indeed, the increase of the density at a given distance is consistent with the finding of an increasing envelope radius and approximately constant average density for the envelope (see the two items above).

\section{Density $n_{10 \mathrm{~K}}$ at $10 \mathrm{~K}$ :}

For a smaller sample, formed by low- and intermediate mass protostars only, it is possible to compare the density $n_{10 \mathrm{~K}}$ at $10 \mathrm{~K}$, which is an indication of the density of the parental cloud (the gas shielded by UV photons). Figure 2 shows that $n_{10 \mathrm{~K}}$ is between $2 \times 10^{4}$ and $6 \times 10^{5} \mathrm{~cm}^{-3}$. This quantity, however, is not correlated with the source luminosity (varying by three orders of magnitude). Therefore there is no evidence that the outer density plays a large role in the determination of the final star mass, a rather important and surprising result, which needs further confirmation.

\section{Summary:}

To summarise, the major result of this section is that the protostar luminosity (namely the mass of the final star) seems preferentially linked to the size (or mass) of the envelope, rather than to the parental cloud density, and that most of the envelope ends up having a centrally condensed, free-fall density distribution. Furthermore, and maybe even more important, there is a continuity in the parameters of the envelopes, going from low- to high-mass protostars. It appears that there is no important difference to the trigger or process of star formation for these two mass regimes. The intermediate mass protostars have allowed a bridge between the low- and high-mass sources, with no apparent observational discontinuity. However, one has to keep in mind that these results are based on single dish observations and are therefore driven mainly by the outer region of the envelopes $\left(z 10^{\prime \prime}\right)$. A more accurate analysis of the smaller scale structure ( cavities, density power law index changes...) will require interferometric observations.

\subsection{The problem of the underestimated $24 \mu \mathrm{m}$ flux}

As mentioned in the previous sections, our modelling fails to reproduce the observed flux at $24 \mu \mathrm{m}$ by several orders of magnitude. This is certainly not due to a numerical problem in the computation but is rather a real problem: our model misses some key element. A possibility evoked in the literature is the presence of a large spherical cavity within the envelope (Jørgensen et al. 2005) which could significantly reduce the optical thickness at $24 \mu \mathrm{m}$.

To check this hypothesis, we carried out a few tests using Cep E-mm as a representative case. The results of the tests are shown in Fig. 3. First, we added a 1800 AU radius cavity to the best-fit model of Table 4 . As expected, the emission at $24 \mu \mathrm{m}$ is increased by several orders of magnitude. However, this model badly fails to reproduce the brightness profiles (as shown in the figure). When a correct procedure is carried out that takes into account the variation in the brightness profiles, namely a minimisation of the $\chi 2$ varying all the envelope parameters, the situation returns to the original (best-fit) underestimation of the $24 \mu \mathrm{m}$ flux. In fact, the introduction of a cavity leads to an increase of the density power law index, $\alpha$, to compensate for the resulting flattening of the model brightness profiles. As a result, the mass found in the outer envelope is lower so that the the overall dust optical depth must be increased to reproduce the integrated submillimetre fluxes. The dust opacity at $24 \mu \mathrm{m}$ again becomes large and very little emission is able to escape at these wavelengths. These tests suggest that to solve the $24 \mu \mathrm{m}$ flux problem it is necessary to have a low-opacity escape route for the $24 \mu \mathrm{m}$ photons and a thick enough envelope to fit the brightness profiles: a simple cavity does not suffice.

Larsson et al. $(2000,2002)$ similarly found that the $24 \mu \mathrm{m}$ flux is underestimated toward Serpens-FIRS1, using different tools and observations. In their first study, Larsson et al. used a $1 \mathrm{D}$ radiative transfer code assuming a spherical envelope model with a single power law density to reproduce the SED (similar to our approach). Their model underestimates the observed flux near $24 \mu \mathrm{m}$ by several orders of magnitude. In their second study, they modelled the envelope with a $2 \mathrm{D}$ radiative transfer code, including a biconical cavity. This model reproduces fairly well the SED from the mid-IR to millimetre wavelengths, but again underestimates the observed flux at $24 \mu \mathrm{m}$ by about a factor $3-10$. The biconical cavity treatment alleviates but does not resolve the missing flux problem. Systematic studies of high-mass protostars by Van der Tak et al. (2000) and Williams et al. (2005) have led to similar results. Williams et al. (2005) modelled 36 high-mass protostellar objects at $850 \mu \mathrm{m}$, using DUSTY, as in the present study. The majority of their best-fits fail to reproduce the flux around $24 \mu \mathrm{m}$. They discuss the possibility of the contribution of high accretion rates, which would significantly increase the near-IR flux density (Osorio et al. 1999). Another contribution could come from the presence of circumstellar disks or the stochastic heating of small grains, which would alter the emission of the envelope and produce more short wavelength photons (e.g. Sellgren et al. 1983; Draine \& Li 2001). However, the envelope is optically thick at these wavelengths, making the emission very sensitive to deviations from the assumed spherical shape. These additional processes still require a low-opacity escape route to exists (also mentioned by Van der Tak et al. 2000) to explain the missing $24 \mu \mathrm{m}$ flux, for example a biconical cavity excavated by the outflow. Note that all the intermediate mass protostars studied here are associated with outflows, except OMC2-FIR4 for which the model indeed fits the observed flux at $24 \mu \mathrm{m}$ (see Crimier et al. 2009). Finally, Van Der Tak et al. 
N. Crimier et al.: The dust physical structure of an Intermediate mass protostars sample

Table 6. Linear correlation coefficients and probability for a chance correlation between the pair of parameters plotted in Figs. 1 and 2.

\begin{tabular}{ccccc}
\hline \hline $\begin{array}{c}\text { Physical parameter } \\
\text { vs. } \log \left(L_{\odot}\right)\end{array}$ & $\begin{array}{c}\text { Linear correlation } \\
\text { coefficient }\end{array}$ & $\begin{array}{c}\text { Number of } \\
\text { objects }\end{array}$ & $\begin{array}{c}\text { Probability for } \\
\text { a chance correlation }\end{array}$ & $\begin{array}{c}\text { Linear fit } \\
\text { slope }\end{array}$ \\
\hline $\log (\alpha)$ & 0.12 & 107 & 1. & - \\
$\log \left(R_{\text {out }}\right)$ & 0.90 & 60 & $10^{-20}$ & 0.3 \\
$\log \left(M_{\text {env }}\right)$ & 0.96 & 46 & $10^{-22}$ & 1.3 \\
$\log \left(n_{1000 \text { AU }}\right)$ & 0.72 & 56 & $10^{-8}$ & 0.3 \\
$\log (\langle n\rangle)$ & 0.25 & 39 & 0.4 & - \\
$\log \left(n_{10 \mathrm{~K}}\right)$ & -0.26 & 18 & 0.5 & - \\
$\log (\theta)$ & 0.88 & 60 & $10^{-19}$ & $-6 \times 10^{-3}$ \\
\hline
\end{tabular}
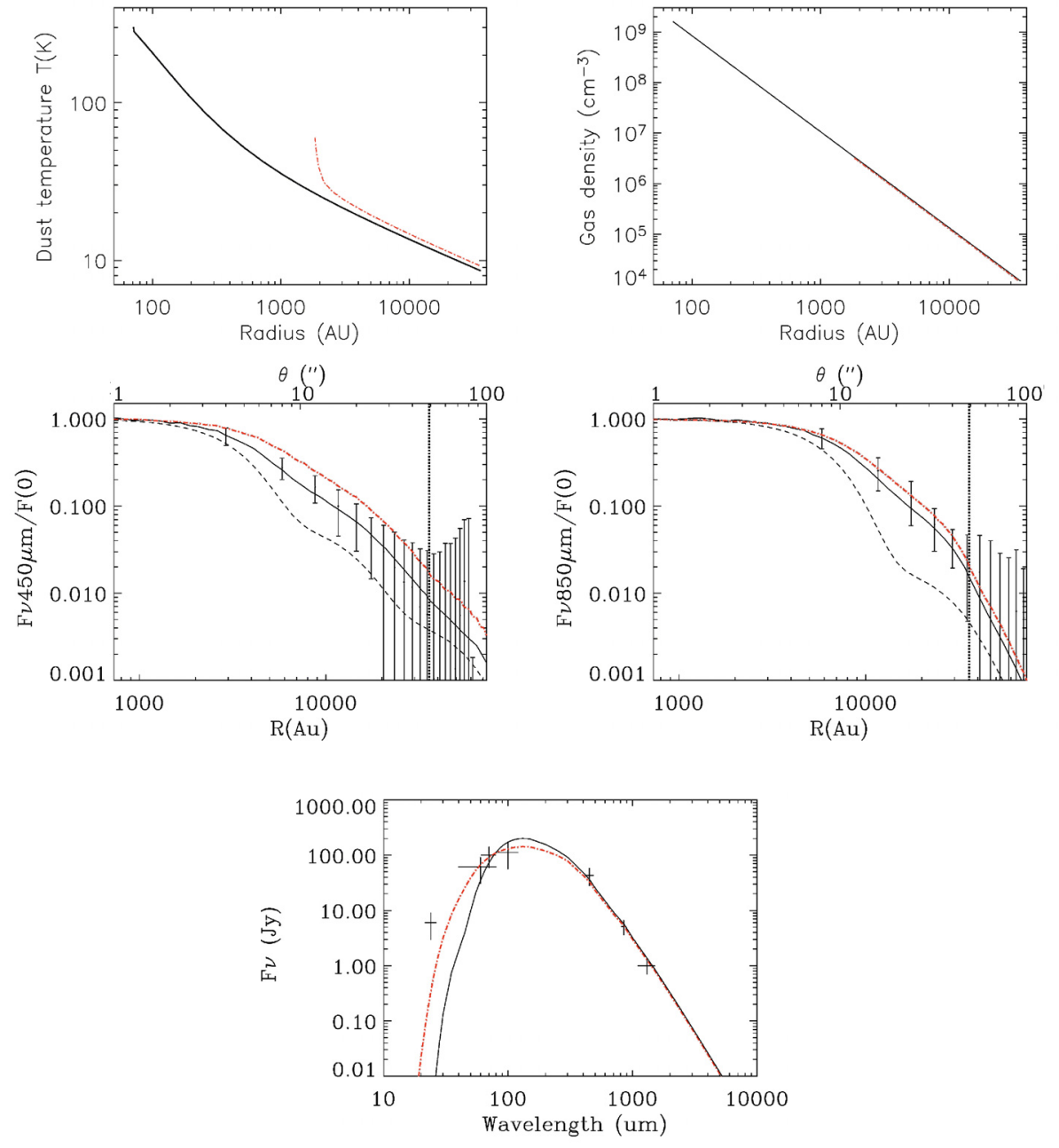

Fig. 3. Cep E-mm physical structure, brightness profiles, and SED from the best-fit model (black solid lines) and after adding an 1800 AU radius cavity to the best-fit model (red dashed-dotted lines). The dust temperature and $\mathrm{H}_{2}$ density profiles are reported in the upper left panel and upper right panel, respectively. The observed brightness profiles at $450 \mu \mathrm{m}, 850 \mu \mathrm{m}$ and the SED, observed and modelled, are shown in the middle left panel, the middle right panel, and the lower panel, respectively. The dashed lines represent the beam pattern of the telescope at $450 \mu \mathrm{m}$ and $850 \mu \mathrm{m}$. The vertical black dotted lines show the outer radius of the best-fit model.

also suggest the possible evaporation of grain ice mantles close to the star, which would decrease the $20 \mu \mathrm{m}$ optical depth by $30 \%$ (Ossenkopf \& Henning 1994) in the $T_{\text {dust }}>100 \mathrm{~K}$ region.

\section{Conclusions}

We have derived the physical structure of the envelopes of five IM protostars, with luminosities between 30 to $1000 L_{\odot}$. The envelope dust density and temperature profiles were determined by means of the $1 \mathrm{D}$ radiative transfer code DUSTY, using all continuum observations from the literature. The analysis assumed that the density profiles follow a single index power law and obtained self-consistently the temperature profile. The bestfit envelope models well reproduce the observations, namely the sub-millimetre radial brightness profiles and the SED between $\sim 60 \mu \mathrm{m}$ and $1.3 \mathrm{~mm}$, for each source. However, the model underestimates the $24 \mu \mathrm{m}$ emission by several orders of magnitude. We ran test models to better understand what ingredient is missing and conclude that a "simple" cavity is not enough to reproduce the $24 \mu \mathrm{m}$ observations. Apparently, the missing ingredient 
is a low-opacity escape route plus a warm dust contribution inside the envelope (circumstellar disc, warm outflow-excavated cavity...).

The gas density and temperature profiles were derived by assuming a constant dust-to-gas ratio and by computing the gas thermal balance at each point within the envelope. Because the gas equilibrium temperature strongly depends on the water abundance in the interiors of the envelopes, we also computed the expected water emission for each source. We found that the gas and dust are thermally coupled across the envelope with differences less than $5 \mathrm{~K}$ in three out of five sources. In IC1396 N BIMA 2 and NGC7129 FIRS 2 the gas is colder than the dust by at most $40 \mathrm{~K}$, in a small region just where the icy mantles are predicted to sublimate. The predicted water line fluxes are consistent with the upper limits derived by the ISO observations.

One of the major goals of the present study was to "use" the IM protostars as a bridge between the low- and high-mass protostars with the hope that this will aid our understanding of the star formation process at either end. When comparing the characteristics derived by the modelling of the envelopes of low-, intermediate, and high-mass protostars, it appears that there is a smooth transition between the various groups. This suggests that there are basically no different triggers or processes between these mass regimes. The power law index $\alpha$ is similar in all three groups of objects. The majority of the sources have $\alpha$ between 1.5 and 2. This is consistent with the theory of isothermal collapse from an initially singular isothermal sphere, the so-called inside-out expansion-wave collapse (Shu 1977). Regardless of the mass group, a few sources have $\alpha$ lower than 1.5 , pointing perhaps to the collapse of an initially logotropic, virialised sphere (Lizano \& Shu 1989, McLaughlin 96, and McLaughlin 97). Finally, the luminosity (mass) of the star depends on the size of the envelope, but does not depend on the density at a given temperature (for example at $10 \mathrm{~K}$ or $100 \mathrm{~K}$ ).

Acknowledgements. We warmly thank Patrick Hennebelle for helpful discussions. One of us (N.Crimier) is supported by a fellowship of the Ministère de l'Enseignement Supérieur et de la Recherche. We acknowledge the financial support by PPF and the Agence Nationale pour la Recherche (ANR), France (contract ANR-08-BLAN-0225). The James Clerk Maxwell Telescope is operated by the Joint Astronomy Centre on behalf of the Science and Technology Facilities Council of the United Kingdom, the Netherlands Organisation for Scientific Research, and the National Research Council of Canada. This paper has been partially supported by MICINN, within the programme CONSOLIDER INGENIO 2010, under grant "Molecular Astrophysics: The Herschel and Alma Era - ASTROMOL" (ref.: CSD2009-00038) Doug Johnstone is supported by a Natural Sciences and Engineering Research Council of Canada (NSERC) Discovery Grant.

\section{Appendix A: Results for individual sources}

\section{A.1. CB3-mm}

\section{A.1.1. Source background}

The CB3 Bok globule is located at $\sim 2.5 \mathrm{kpc}$ (Launhardt \& Henning 1997; Wang et al. 1995) on the near side of the Perseus arm of the Galaxy. Using ISO data, Launhardt et al. (1998) carried out a multi-wavelength study of the CB3 globule and derived a total mass and bolometric luminosity of the order of $400 M_{\odot}$ and $\sim 1000 L_{\odot}$, respectively, for the entire globule. The globule hosts $\sim 40$ NIR sources, 22 of which are likely lowand intermediate mass protostars in different stages of evolution (Launhardt et al. 1998; Yun \& Clemens 1995, 1994). CB3-mm, the brightest millimetre source in the globule, was first detected by Launhardt \& Henning (1997) and subsequently observed in the sub-millimetre by Huard et al. (2000). The high luminosity of the source evaluated by Launhardt \& Henning (1997), $L \sim 900 L_{\odot}$, suggests that CB3-mm is an intermediate mass object. A recent interferometric study by Fuente et al. (2007) showed that CB3-mm contains two compact cores at $3 \mathrm{~mm}$ separated by $0.3 \mathrm{pc}\left(\sim 0.43^{\prime \prime}\right)$. Yun \& Clemens (1994) also detected a molecular bipolar outflow in $\mathrm{CO}$, elongated in the northeastsouthwest direction, associated with $\mathrm{H}_{2} \mathrm{O}$ masers (de GregorioMonsalvo et al. 2006). This outflow has been mapped in various molecular lines by Codella \& Bachiller (1999), who concluded that it originates from CB3-mm. The same authors concluded that CB3-mm is probably a Class 0 source. In the present study we re-evaluated the luminosity of this object, $L \sim 1000 L_{\odot}$, making $\mathrm{CB} 3-\mathrm{mm}$ an intermediate mass protostar.

\section{A.1.2. Analysis}

The continuum maps used for the CB3-mm analysis are presented in Fig. A.1. The maps at $450 \mu \mathrm{m}$ and $850 \mu \mathrm{m}$ were obtained on 1997 December 18 as a part of project m96bi28 and on 1998 August 10 as a part of project m98bc21, respectively. The flux profiles obtained at each wavelength are shown in Fig. A.2. The Spitzer observations were obtained on the 20th September 2004 as part of the programme "Comparative Study of Galactic and Extragalactic HII Regions" (AOR: 63, PI: James R. Houck). The integrated fluxes used for the analysis are reported in Table 2 and in Fig. A.2. The fluxes at $70 \mu \mathrm{m}, 450 \mu \mathrm{m}$ and $850 \mu \mathrm{m}$ were obtained by integration over a $\sim 50^{\prime \prime}$ radius. The uncertainty ellipse position of the IRAS observations are reported on the $450 \mu \mathrm{m}$ maps in Fig. A.1. Note that the map at $24 \mu \mathrm{m}$ presents two sources separated by $\sim 12^{\prime \prime}$, centred on the sub-millimetre source emission (see Fig. A.1). Therefore, the flux $1.4 \pm 0.8 \mathrm{Jy}$ at $24 \mu \mathrm{m}$ was obtained by adding the integrated flux over the two sources separately. We also report on the SED the lower limits from Plateau de Bure (PdB) interferometric fluxes at $1.3 \mathrm{~mm}$ and $3 \mathrm{~mm}$ (Fuente et al. 2007).

\section{A.1.3. Best-fit}

Table 4 presents the set of parameters $\alpha, Y$, and $\tau_{100}$, which best reproduce the observations, and summarises some relevant physical quantities of the model. Figure A.2 shows the best derived brightness profiles and SED against the observed ones, while Fig. A. 3 shows the $\chi^{2}$ contour plots. The dust density and temperature profiles of the best-fit model are reported in Fig. A.4. Although the observed flux profiles and SED fluxes from $60 \mu \mathrm{m}$ to $850 \mu \mathrm{m}$ are well reproduced by the model, the observed flux at $24 \mu \mathrm{m}$ is underestimated by about two orders of magnitude. The model agrees well with the lower limits from Plateau de Bure interferometric fluxes at $1.3 \mathrm{~mm}$ and $3 \mathrm{~mm}$ (Fuente et al. 2007), however.

The best-fit model, obtained with an $\alpha$ of 2.2 , is the steepest among the five sources modelled in the paper and leads to a strong gradient in the density profile, from $\sim 10^{9}$ to $\sim 10^{3} \mathrm{~cm}^{-3}$ with a density and radius at $100 \mathrm{~K}$ of $7.5 \times 10^{7} \mathrm{~cm}^{-3}$ and $700 \mathrm{AU}$, respectively.

Although CB3-mm is the largest and brightest of the five sources investigated in this paper, its distance of $2500 \mathrm{pc}$ makes it the least resolved source. Indeed, the brightness profiles at each wavelength presented in Fig. A.2 are close to the beam patterns of the telescope, showing that the source is barely resolved. Consequently, the value of the envelope radius $r_{\text {out }}$ suffers a relatively large uncertainty. Considering the $\chi_{\text {red }}^{2}$ contour at $10 \%$ of 
N. Crimier et al.: The dust physical structure of an Intermediate mass protostars sample

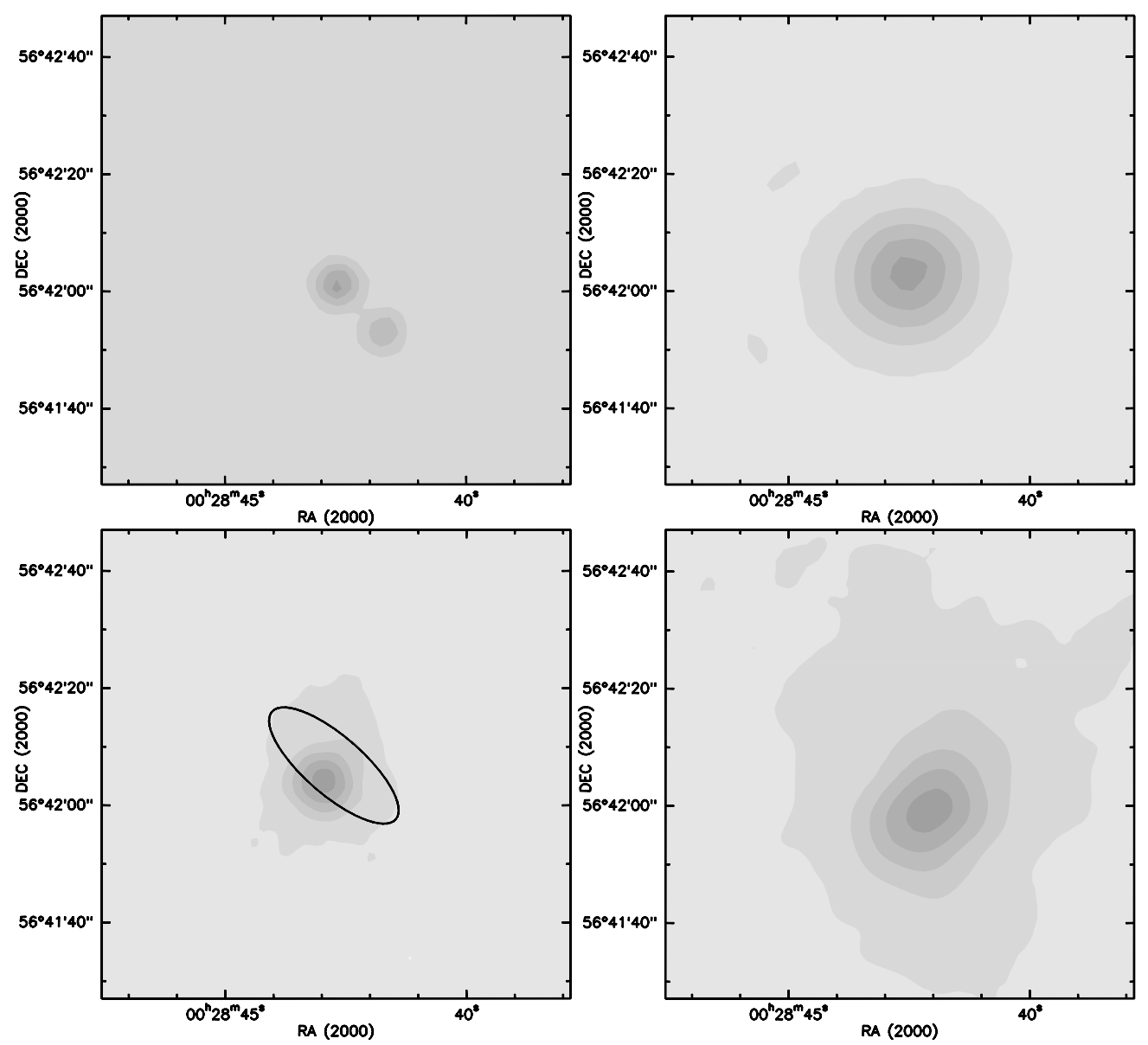

Fig. A.1. Continuum emission maps around CB3-mm at $24 \mu \mathrm{m}$ (upper left panel), $70 \mu \mathrm{m}$ (upper right panel), $450 \mu \mathrm{m}$ (lower left panel), and $850 \mu \mathrm{m}$ (lower right panel). The grey scale contours mark the continuum flux from $10 \%$ to $90 \%$ of the peak emission by steps of $20 \%$. The uncertainty ellipse position of the IRAS observations toward CB3-mm are represented by the ellipse in the $450 \mu \mathrm{m}$ map.

the minimum $\chi_{\text {red }}^{2}$ (see Fig. A.3) in order to estimate the uncertainty, we obtain $r_{\text {out }}=\left(1.0 \pm_{3.9}^{1.3}\right) \times 10^{5}$ AU. On the contrary, the envelope column density is quite insensitive to $r_{\text {out }}$, due to the high power law index of the density profile. Finally, in minimising the $\chi_{\text {SED }}^{2}$, we also varied the source luminosity from $800 L_{\odot}$ to $1200 L_{\odot}$ and found that the best-fit is obtained with source luminosity equal to $1000 L_{\odot}$.

\section{A.2. Cep E-mm}

\section{A.2.1. Source background}

Located in the Cepheus OB3 association at a distance of $730 \mathrm{pc}$ (Sargent 1977; Crawford \& Barnes 1970), Cepheus E is the second most massive and dense clump of this region (Few \& Cohen 1983). Cepheus E hosts the source IRAS 23011+6126, which is associated with Cep E-mm, catalogued as a Class 0 protostar by Lefloch et al. (1996). Targeted by several continuum and lines studies, Cep E-mm has been observed with IRAS (Palla et al. 1993), IRAM 30m (Lefloch et al. 1996; Chini et al. 2001), SCUBA (Chini et al. 2001), ISO (Froebrich et al. 2003), and Spitzer (Noriega-Crespo et al. 2005). All these studies confirm the Class 0 status of Cep E-mm and constrain the total mass and bolometric luminosity of the source to 7$25 M_{\odot}$ and $\sim 80-120 L_{\odot}$, respectively. Finally, a bipolar molecular outflow, first reported by Fukui et al. (1989), is associated with Cep E-mm. The properties of the outflow have been thoroughly analysed by Eislöffel et al. (1996), Ayala et al. (2000),
Moro-Martín et al. (2001), and Smith et al. (2003). The $\mathrm{H}_{2}$ and [FeII] study by Eislöffel et al. (1996) shows a quadrapolar outflow morphology, suggesting that the driving source is a binary. This outflow morphology has been confirmed by sub-mm and near-IR observations (Ladd \& Hodapp 1997), and by Spitzer observations (Noriega-Crespo et al. 2005).

\section{A.2.2. Analysis}

The continuum maps used for the Cep E-mm analysis are presented in Fig. A.5. The maps at $450 \mu \mathrm{m}$ and $850 \mu \mathrm{m}$ were obtained in August 1997 as a part of the project m97bu87 and the observations are described in detail by Chini et al. (2001). The flux profiles obtained at each wavelength are shown in Fig. A.6. The Spitzer observations were obtained on the 2003 September 29 as part of the programme "MIPS/IRAC imaging of protostellar jet HH 212" (AOR: 1063, PI: Alberto Noriega-Crespo) and are described in detail by Noriega-Crespo \& Garnavich (2001). The integrated fluxes at $450 \mu \mathrm{m}, 850 \mu \mathrm{m}$, and $1300 \mu \mathrm{m}$ were retrieved from the literature (Chini et al. 2001). The uncertainty ellipse position of the IRAS observation is reported on the $450 \mu \mathrm{m}$ map in Fig. A.5. The fluxes at $24 \mu \mathrm{m}$ and $70 \mu \mathrm{m}$ were obtained by integration over a $\sim 30^{\prime \prime}$ and $\sim 60^{\prime \prime}$ radius, respectively. Note that to compute the flux uncertainties, we varied the integration radius of about $50 \%$ and found variations of $\lesssim 30 \%$. The integrated fluxes used for the analysis are reported in Table 2 and in Fig. A.6. 

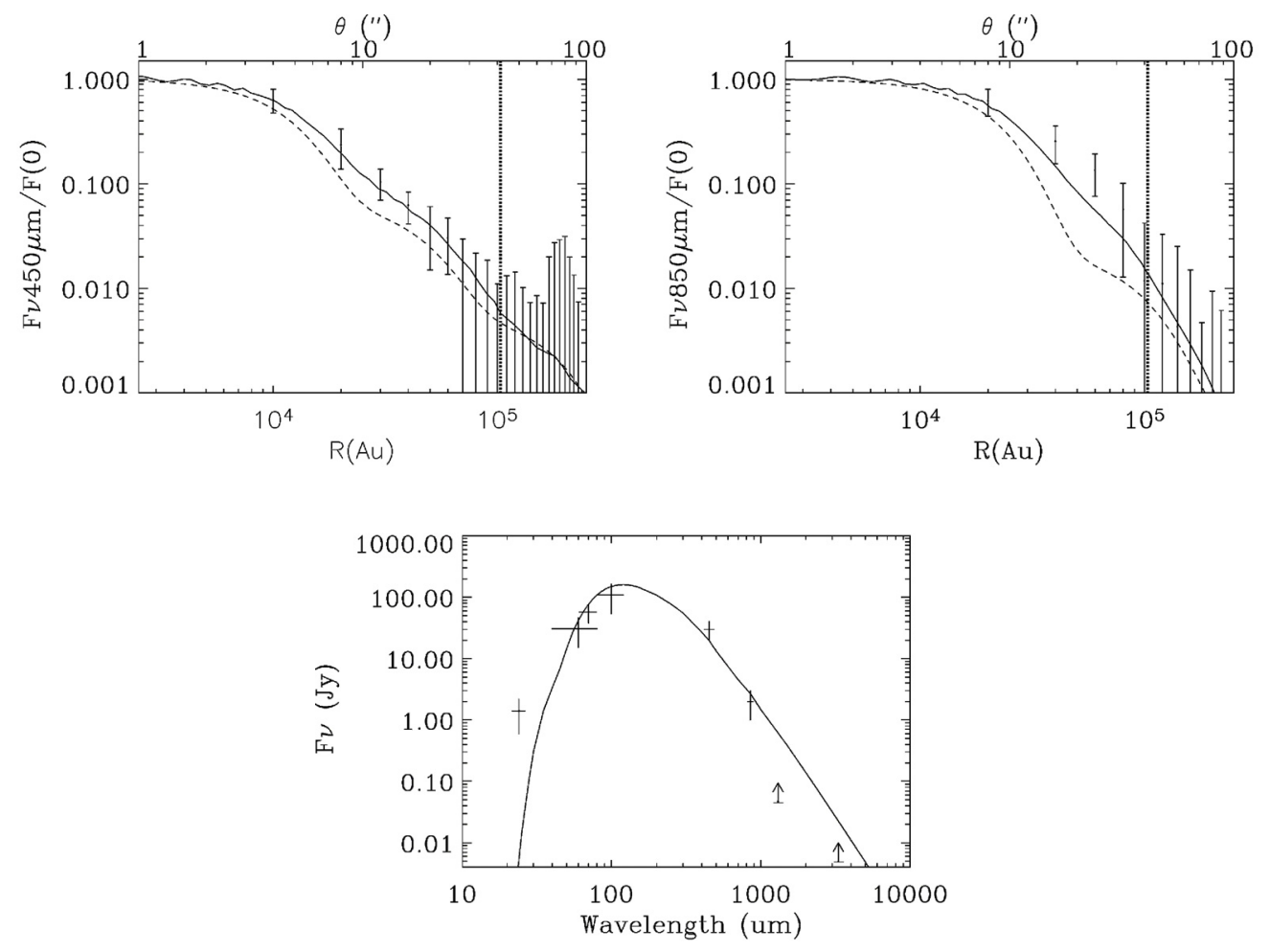

Fig. A.2. Observed brightness profiles at $450 \mu \mathrm{m}$ (upper left panel), $850 \mu \mathrm{m}$ (upper right panel) and SED (lower centred panel) toward CB3-mm. The solid lines report the best-fit model. The dashed lines represent the beam pattern of the telescope at $450 \mu \mathrm{m}$ and $850 \mu \mathrm{m}$. The vertical dotted lines show the outer radius of the best-fit model (Table 4). The lower limits from Plateau de Bure (PdB) interferometric fluxes at $1.3 \mathrm{~mm}$ and $3 \mathrm{~mm}$ (Fuente et al. 2007) are also reported on the SED.

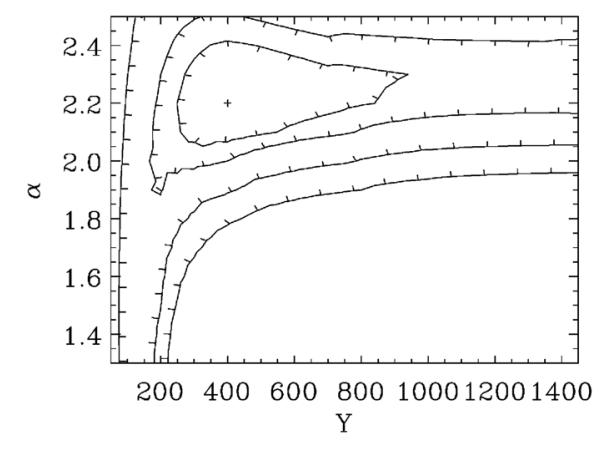

Fig. A.3. $\chi_{\text {red }}^{2}$ contour plots $(Y, \alpha)$ for the CB3-mm model. In these computations, $\tau_{100}$ is equal to 5.8. The contours show the loci of the $\chi_{\text {red }}^{2}$ values equal to $1.1,1.2,1.5$ and 2 times the minimum $\chi_{\text {red }}^{2}(10,20,50$ and $100 \%)$. The cross shows the position of the best-fit.

\section{A.2.3. Best-fit}

Table 4 presents the set of parameters $\alpha$, Y, and $\tau_{100}$, which best reproduce the observations, and summarises some relevant physical quantities of the model. Figure A.6 shows the derived brightness profiles and SED against the observations. The dust density and temperature profiles of the best-fit model are reported in Fig. A.7. Although the observed flux profiles and SED fluxes from $60 \mu \mathrm{m}$ to $1300 \mu \mathrm{m}$ are well reproduced by the model, the observed flux at $24 \mu \mathrm{m}$ is underestimated by about $\sim 3$ orders of magnitude. Similarly to CB3-mm, the envelope model derived for Cep E-mm with an $\alpha$ of 1.9 is very peaked, leading to a strong gradient in the density profile, from $\sim 10^{9}$ to $\sim 10^{4} \mathrm{~cm}^{-3}$ with a density and radius at $100 \mathrm{~K}$ of $2.0 \times 10^{8} \mathrm{~cm}^{-3}$ and $223 \mathrm{AU}$, respectively. Considering the $\chi_{\text {red }}^{2}$ contour at $10 \%$ of the minimum $\chi_{\text {red }}^{2}$ in order to estimate the uncertainty, we obtained $r_{\text {out }}=(3.6 \pm 0.7) \times 10^{4}$ AU. Finally, in minimising the $\chi_{\mathrm{SED}}^{2}$, we also varied the source luminosity from $70 L_{\odot}$ to $130 L_{\odot}$ and found the best-fit for a source luminosity equal to $100 L_{\odot}$.

\section{A.3. IC1396 N BIMA 2}

\section{A.3.1. Source background}

IC1396 N is a bright globule located 750 pc (Matthews 1979) from the Sun, near the border of the IC1396 extended HII region (Osterbrock 1957; Weikard et al. 1996) and at a projected distance of 11 pc north of the O6.5 star HD 206267 which ionises the region. IC1396 N is associated with the source IRAS21391+5802. Its strong submillimetre and millimetre continuum emission (Wilking et al. 1993; Sugitani et al. 2000; Codella et al. 2001), high-density gas (Serabyn et al. 1993; Cesaroni et al. 1999; Codella et al. 2001; Beltrán et al. 2004), and water maser emission (Felli et al. 1992; Tofani et al. 1995; Patel et al. 2000) reveal that IC1396 N is an active site of star formation. The bolometric luminosity is estimated to range from $235 L_{\odot}$ (Saraceno et al. 1996) to $440 L_{\odot}$ (Sugitani et al. 2000).

Using BIMA interferometric millimetre observations, Beltrán et al. (2002) detected three sources (BIMA 1, BIMA 2 and BIMA 3) deeply embedded in the globule. BIMA 2 has the strongest millimetre emission and is also the most massive object. The authors concluded that BIMA 2 is most likely an IM protostar, while BIMA 1, and BIMA 3, located at $\sim 15^{\prime \prime}$ west and south-east respectively, are less massive and/or more evolved. The recent studies by Neri et al. (2007) and Fuente et al. (2007), 
N. Crimier et al.: The dust physical structure of an Intermediate mass protostars sample
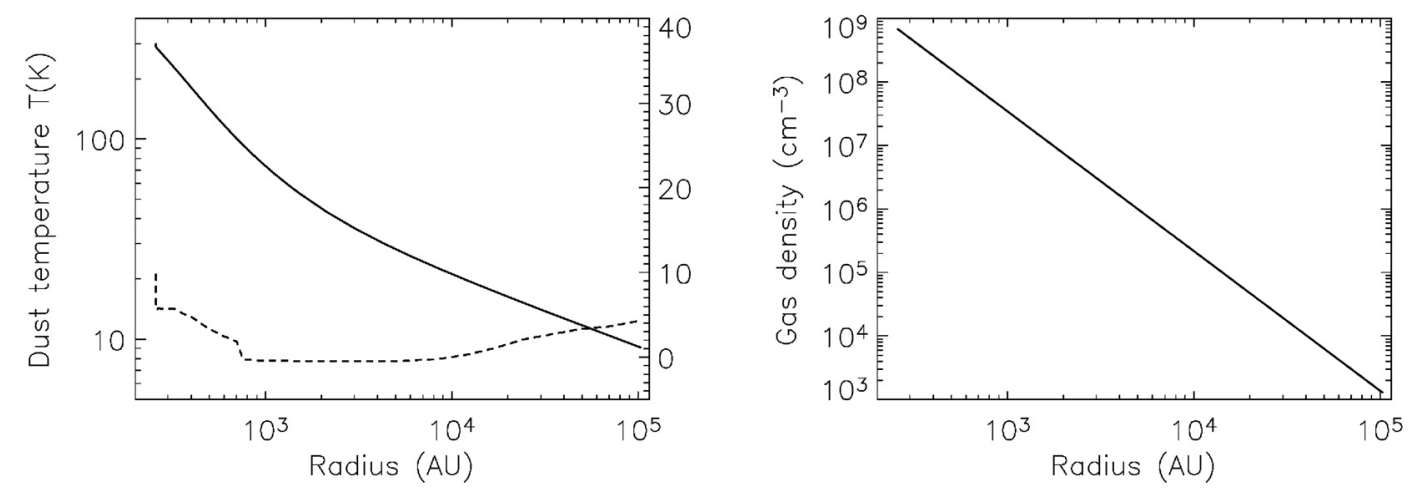

Fig. A.4. CB3-mm dust temperature (left panel) and $\mathrm{H}_{2}$ density (right panel) profiles from the best-fit model. The dashed line represents the

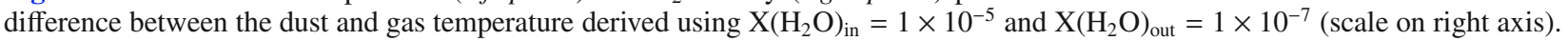

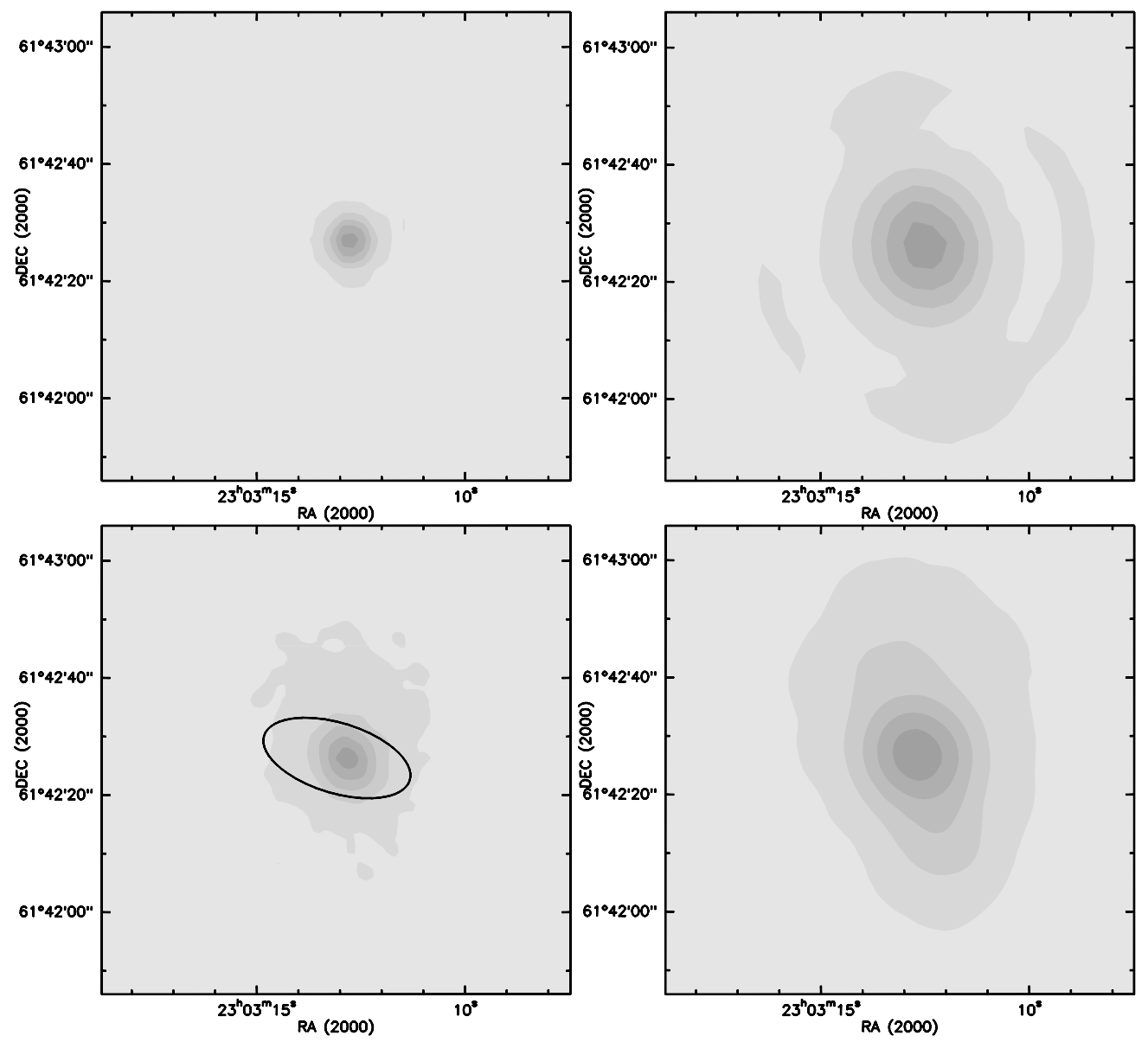

Fig. A.5. Continuum emission maps around Cep E-mm at $24 \mu \mathrm{m}$ (upper left panel), $70 \mu \mathrm{m}$ (upper right panel), $450 \mu \mathrm{m}$ (lower left panel), and $850 \mu \mathrm{m}$ (lower right panel). The grey scale contours mark the continuum flux from $10 \%$ to $90 \%$ of the peak emission in steps of $20 \%$. The uncertainty ellipse position of the IRAS observation toward Cep E-mm is reported on the $450 \mu \mathrm{m}$ map.

using Plateau de Bure interferometric observations, provide a detailed study of BIMA 2 and showed that BIMA 2 contains at least three dense cores separated by $\sim 1^{\prime \prime}$.

Finally, the region presents an extended ( 3 arcmin) CO bipolar outflow (Sugitani et al. 1989) which has been mapped by Codella et al. (2001). The study by Nisini et al. (2001), revealed several $\mathrm{H}_{2}$ jets inside the region. A more recent analysis (Beltrán et al. 2002, 2004) shows that BIMA 1 and
BIMA 2 are associated with north-south and east-west bipolar molecular outflows, respectively.

\section{A.3.2. Analysis}

The continuum maps used for the IC1396 N BIMA 2 analysis are presented in Fig. A.8. The SCUBA maps were obtained 

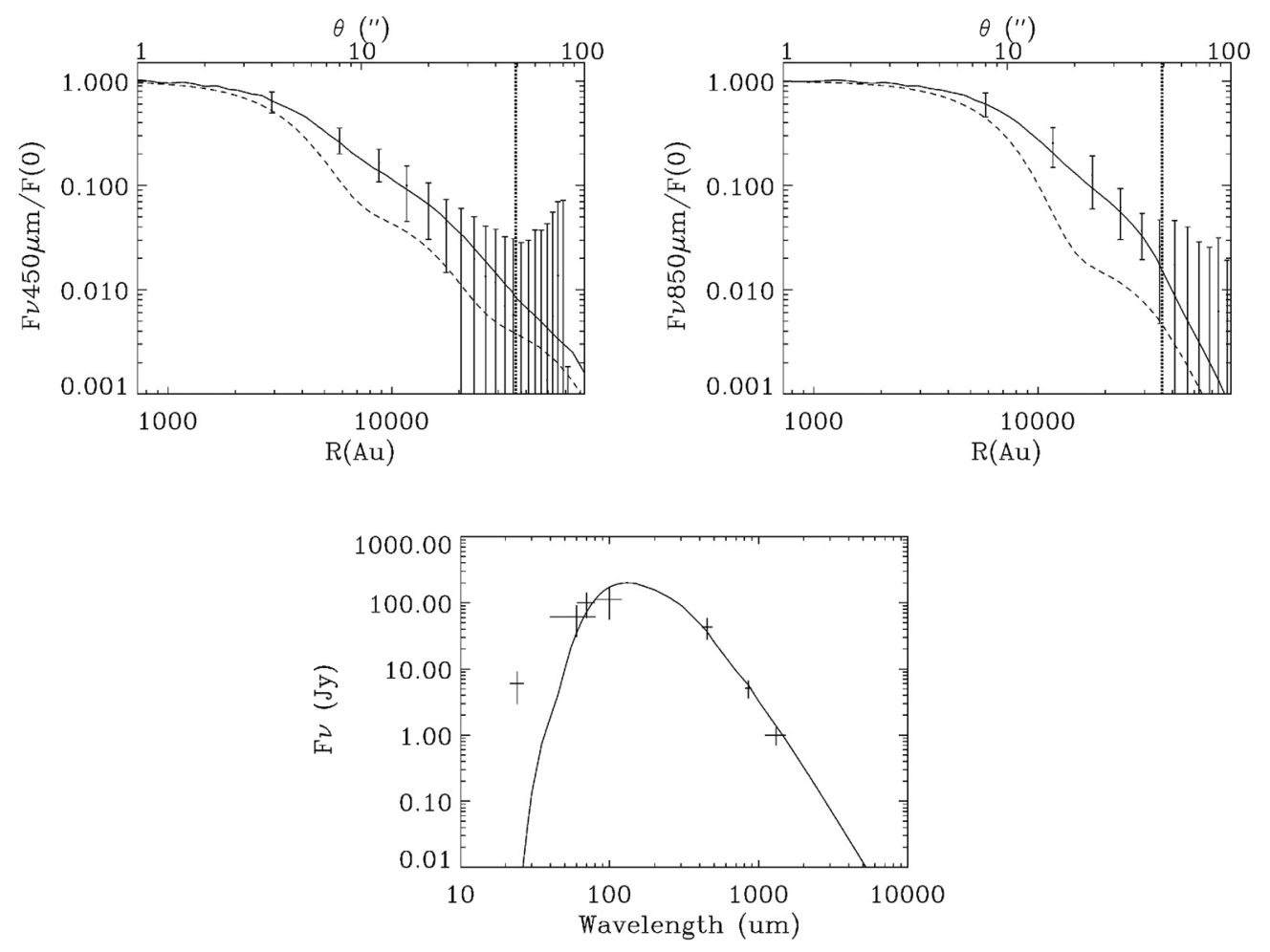

Fig. A.6. Observed brightness profiles at $450 \mu \mathrm{m}$ (upper left panel), $850 \mu \mathrm{m}$ (upper right panel), and the SED (lower centred panel) toward Cep E-mm. The solid lines report the best-fit model (Table 4). The dashed lines represent the beam pattern of the telescope at $450 \mu \mathrm{m}$ and $850 \mu \mathrm{m}$. The vertical dotted lines show the outer radius of the best-fit model.
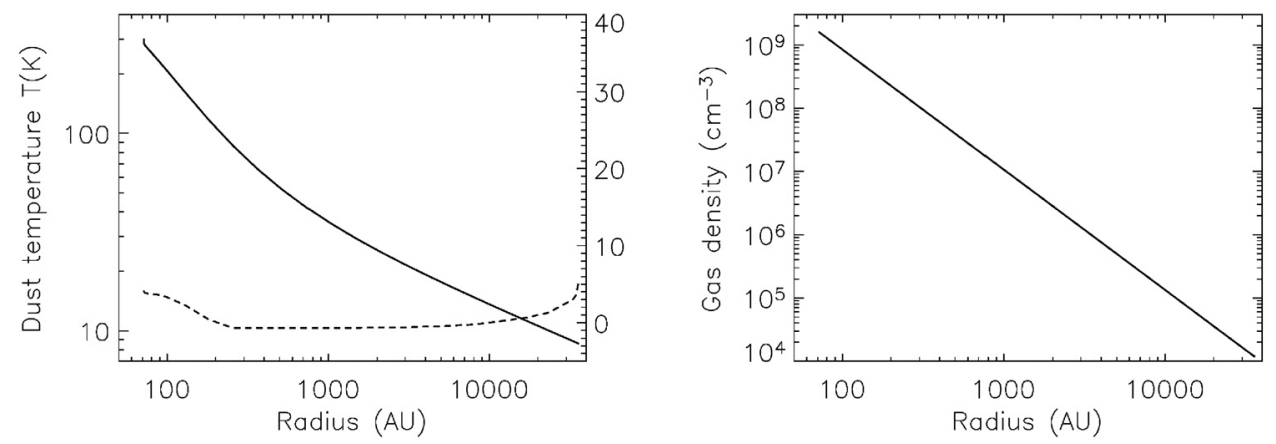

Fig. A.7. Cep E-mm dust temperature (left panel) and $\mathrm{H}_{2}$ density (right panel) profiles from the best-fit model. The dashed line represents the

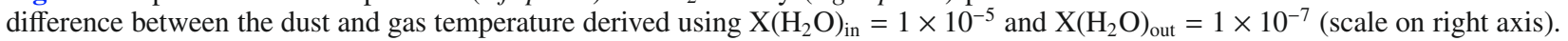

in March 2002 as a part of the project m02au07. The brightness profiles (see Fig. A.9) are derived excluding the regions contaminated by the presence of BIMA 1 and BIMA 3 (dashed regions in Fig. A.8). The Spitzer observations were obtained in October 2004 as part of the programme "Star Formation in Bright Rimmed Clouds" (AOR: 202, PI: Giovanni Fazio). The integrated fluxes used for the analysis are reported in Table 2 and in Fig. A.9. The integrated fluxes at $450 \mu \mathrm{m}$ and $850 \mu \mathrm{m}$ were obtained by integration over a $\sim 35^{\prime \prime}$. We varied the integration radius from $20^{\prime \prime}$ to $90^{\prime \prime}$, and found variations on the fluxes $\lesssim 30 \%$. The large uncertainty in the integrated flux at $24 \mu \mathrm{m}$ is due to the proximity of BIMA 3 (see $24 \mu \mathrm{m}$ map in Fig. A.8). Note that due to the large IRAS beams at $60 \mu \mathrm{m}$ and $100 \mu \mathrm{m}$, BIMA 3 introduces significant spurious signal. We thus used the IRAS and $70 \mu \mathrm{m}$ Spitzer fluxes as upper limits on the SED (Fig A.9).

\section{A.3.3. Best-fit}

Table 4 presents the set of parameters $\alpha$, Y, and $\tau_{100}$, which best reproduce the observations, and summarises some relevant physical quantities of the model. Figure A.9 shows the relevant derived brightness profiles and SED against the observations. The dust density and temperature profiles of the best-fit model are reported in Fig. A.10. The observed flux profiles and SED fluxes at $450 \mu \mathrm{m}$ and $850 \mu \mathrm{m}$ are well reproduced by the model. Considering the IRAS and $70 \mu \mathrm{m}$ Spitzer fluxes as upper limits (see Sect. A.3.2), the minimum $\chi_{\text {SED }}^{2}$ is obtained for a source luminosity equal to $150 L_{\odot}$, similar to the value suggested by Beltrán et al. (2002). The observed flux at $24 \mu \mathrm{m}$ is underestimated by about one order of magnitude. Considering the $\chi_{\text {red }}^{2}$ contour at $10 \%$ of the minimum $\chi_{\text {red }}^{2}$ to estimate the uncertainty, 
N. Crimier et al.: The dust physical structure of an Intermediate mass protostars sample

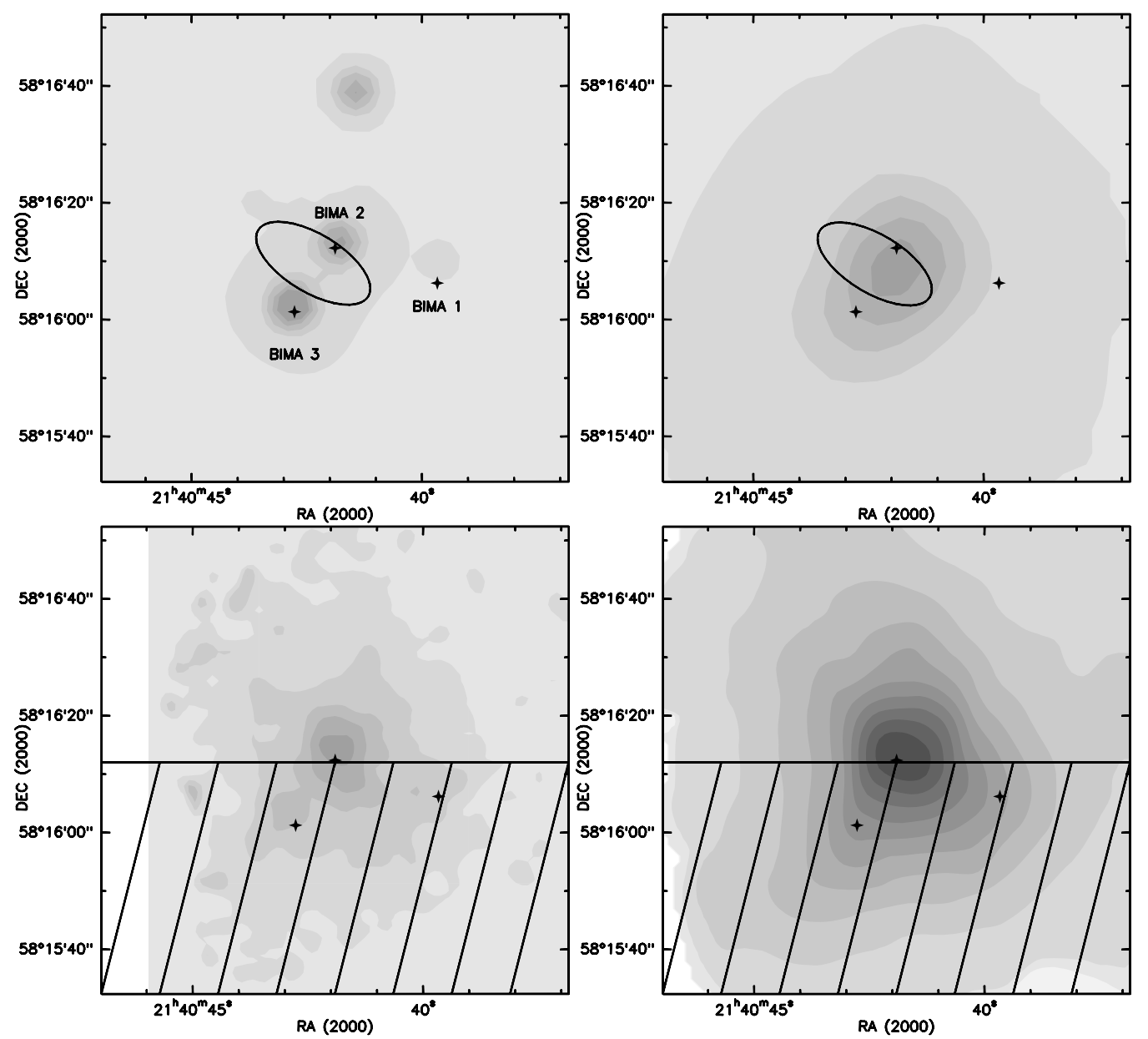

Fig. A.8. Continuum emission maps around IC1396 N BIMA 2 at $24 \mu \mathrm{m}$ (upper left panel), $70 \mu \mathrm{m}$ (upper right panel), $450 \mu \mathrm{m}$ (lower left panel), and $850 \mu \mathrm{m}$ (lower right panel). The grey scale contours mark the continuum flux from $10 \%$ to $90 \%$ of the peak emission by steps of $20 \%$. The positions of BIMA 1, BIMA 2 and BIMA 3, as well as the uncertainty ellipse position of the IRAS observations are reported on the $24 \mu \mathrm{m}$ map. The dashed regions on the $450 \mu \mathrm{m}$ and $850 \mu \mathrm{m}$ maps mark the regions excluded to derive the flux profiles.

we obtained $r_{\text {out }}=(3.0 \pm 0.6) \times 10^{4}$ AU. Note that we obtain similar results without subtracting the dashed region of Fig. A.8, confirming the weak contribution of BIMA 1 and 3 to the submillimetre emission.

Finally, contrary to the previous sources, the gas temperature profile obtained for IC1396 N BIMA 2 is slightly decoupled from the dust temperature in the inner region (see Fig. A.10). The origin of this thermal decoupling is discussed in detail in Crimier et al. (2009). Briefly, the decoupling is due to a competition between the water abundance (the main coolant of the gas in the inner region) and the dust density, which mainly regulates the balance energy in the inner part of the envelope. This thermal decoupling is stronger in IC1396 N BIMA 2 (and NGC7129 FIRS 2) than in the other sources because of the lower density in the inner region. The thermal decoupling reaches a maximum value of about $40 \mathrm{~K}\left(\sim 15 \%\right.$ of $\left.T_{\text {dust }}\right)$ for an abundance of $\mathrm{X}\left(\mathrm{H}_{2} \mathrm{O}\right)_{\text {in }}=1 \times 10^{-5}$ and about $10 \mathrm{~K}$ for an abundance of $\mathrm{X}\left(\mathrm{H}_{2} \mathrm{O}\right)_{\text {in }}=1 \times 10^{-6}$.

\section{A.4. NGC7129 FIRS 2}

\section{A.4.1. Source background}

NGC7129 is a reflection nebula located in a complex and active molecular cloud (Hartigan \& Lada 1985; Miranda et al. 1993) and estimated to be at a distance of $1250 \pm 50 \mathrm{pc}$ (Shevchenko \& Yakubov 1989) from the Sun. The region contains several Herbig AeBe stars, which illuminate the nebula. NGC7129 FIRS 2 has been detected in the far-infrared by Bechis et al. (1978) and Harvey et al. (1984). FIRS 2 is not detected at optical or nearinfrared wavelengths. Its position coincides with a ${ }^{13} \mathrm{CO}$ column density peak (Bechis et al. 1978) and a high-density $\mathrm{NH}_{3}$ cloudlet (Guesten \& Marcaide 1986). It is also close to an $\mathrm{H}_{2} \mathrm{O}$ maser (Rodriguez et al. 1980). NGC7129 FIRS 2 has been classified as an IM Class 0 source by Eiroa et al. (1998), who carried out a multi-wavelength study of the continuum emission from $25 \mu \mathrm{m}$ to $2000 \mu \mathrm{m}$. These authors estimate a total mass and bolometric luminosity of $\sim 6 M_{\odot}$ and $\sim 430 L_{\odot}$, respectively. In addition, Edwards \& Snell (1983) detected a bipolar CO outflow associated with FIRS 2. The interferometric study of Fuente et al. (2001) has confirmed this bipolar outflow and pointed out a quadrapolar morphology to the flow. This quadrapolar morphology seems to be due to the superposition of two flows, FIRS 2out 1 and FIRS 2-out 2, likely associated with FIRS 2 and a more evolved star (FIRS 2-IR), respectively. Finally, Fuente et al. $(2005 a, b)$ carried out an extensive chemical study of FIRS 2 providing the first detection of a hot core in an IM Class 0. Based on all these studies, FIRS 2 is considered the youngest IM object known at present. 

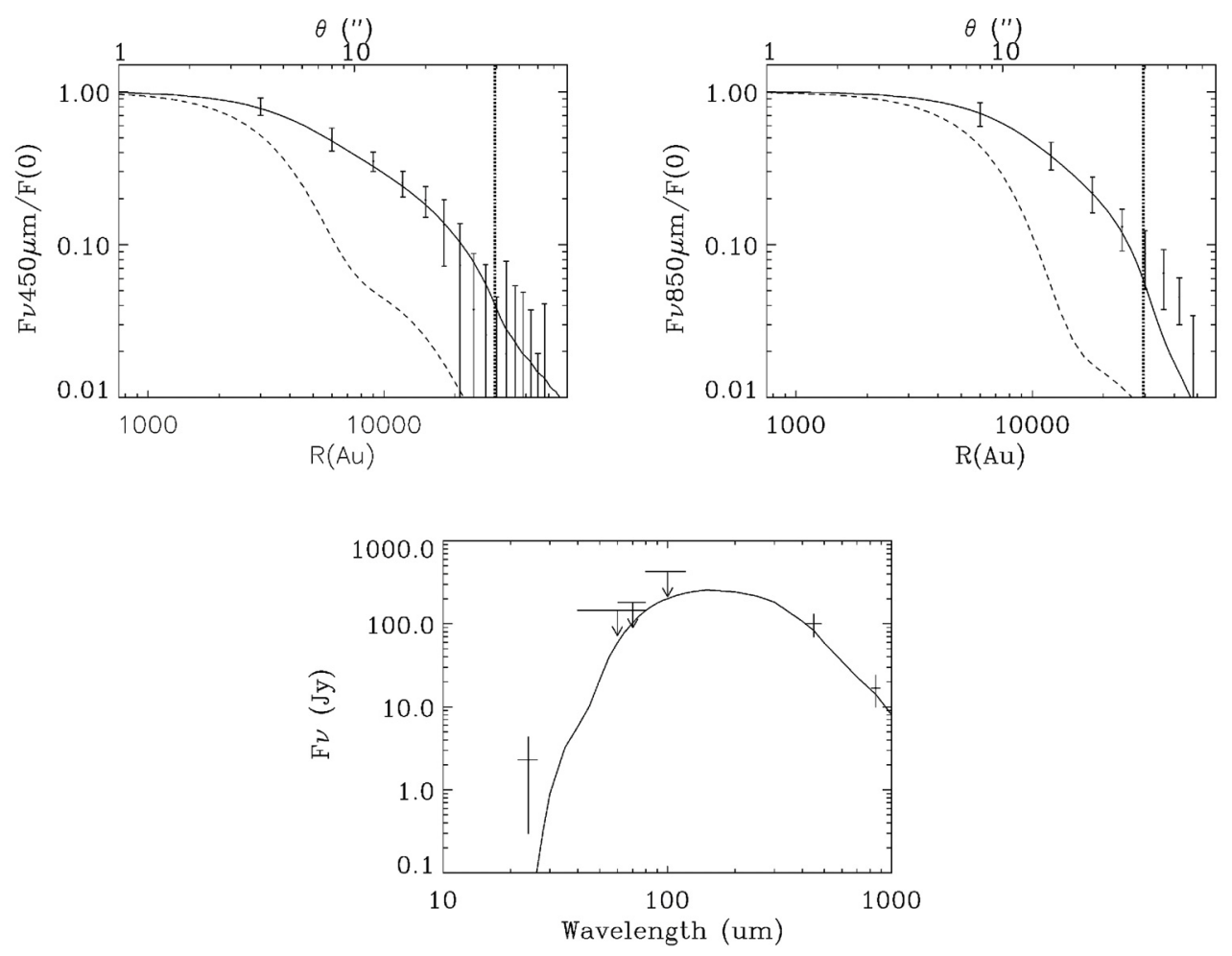

Fig. A.9. Observed brightness profiles at $450 \mu \mathrm{m}$ (upper left panel), $850 \mu \mathrm{m}$ (upper right panel) and the SED (lower centred panel) toward IC1396 N BIMA 2. The solid lines report the best-fit model (Table 4). The dashed lines represent the beam pattern of the telescope at $450 \mu \mathrm{m}$ and $850 \mu \mathrm{m}$. The vertical dotted lines show the outer radius of the best-fit model. The IRAS and $70 \mu \mathrm{m}$ Spitzer fluxes are used as upper limits on the SED.
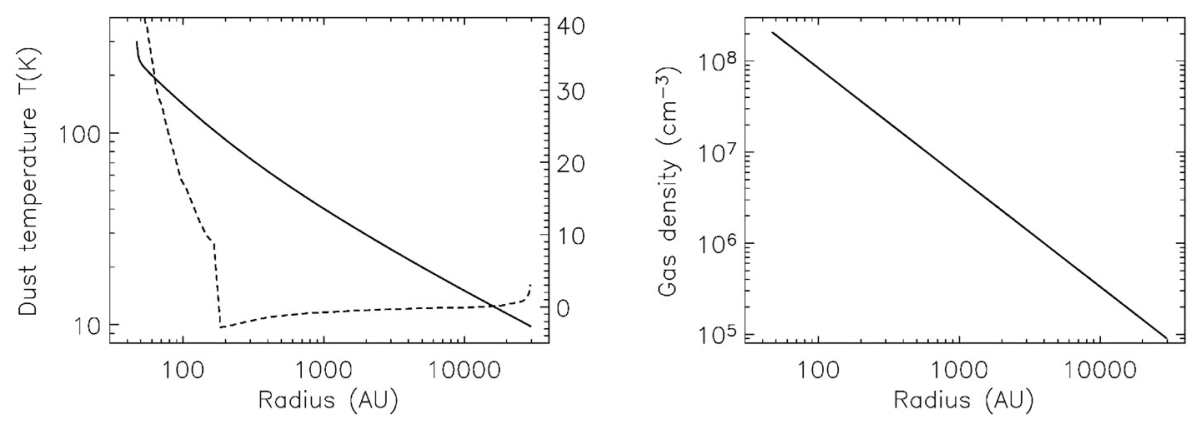

Fig. A.10. IC1396 N BIMA 2 dust temperature (left panel) and $\mathrm{H}_{2}$ density (right panel) profiles from the best-fit model. The dashed line represents the difference between the dust temperature and the gas temperature derived using $\mathrm{X}\left(\mathrm{H}_{2} \mathrm{O}\right)_{\text {in }}=1 \times 10^{-5}$ and $\mathrm{X}\left(\mathrm{H}_{2} \mathrm{O}\right)_{\text {out }}=1 \times 10^{-7}(\mathrm{scale}$ on right axis).

\section{A.4.2. Analysis}

All the data and continuum maps used for the NGC7129 FIRS 2 analysis are reported in Table 2 and Fig. A.11. The maps at $450 \mu \mathrm{m}$ and $850 \mu \mathrm{m}$ were obtained on the 1998 August 26 as a part of the project M98BU24. We also used the flux profile and the integrated flux at $1300 \mu \mathrm{m}$ extracted from the single dish map at $1300 \mu \mathrm{m}$, presented in Fuente et al. (2001). Following Greve et al. (1998), the beam at $1300 \mu \mathrm{m}$ is assumed to be a combination of three Gaussian curves, with $H P B W \mathrm{~s}$ of $11^{\prime \prime}, 125^{\prime \prime}$, and 180", with amplitude ratios of $0.975,0.005$, and 0.001 , respectively. The Spitzer observations were obtained on the 2003 September 19 as part of the programme "Protostars and Proto-Brown Dwarfs in a Nearby Dark Cloud" (AOR: 34,
PI: Tom Megeath). The integrated fluxes reported in Table 2 and in Fig. A.12 were obtained by integration over a $\sim 40^{\prime \prime}$ radius. The IRAS integrated fluxes at $60 \mu \mathrm{m}$ and $100 \mu \mathrm{m}$ were taken from Eiroa et al. (1998).

\section{A.4.3. Best-fit}

Table 4 presents the set of parameters $\alpha$, Y, and $\tau_{100}$, which best reproduce the observations, and summarises some relevant physical quantities of the model. Figure A.12 shows the relevant derived brightness profiles and SED against the observations. The dust density and temperature profiles of the best-fit model are 
N. Crimier et al.: The dust physical structure of an Intermediate mass protostars sample
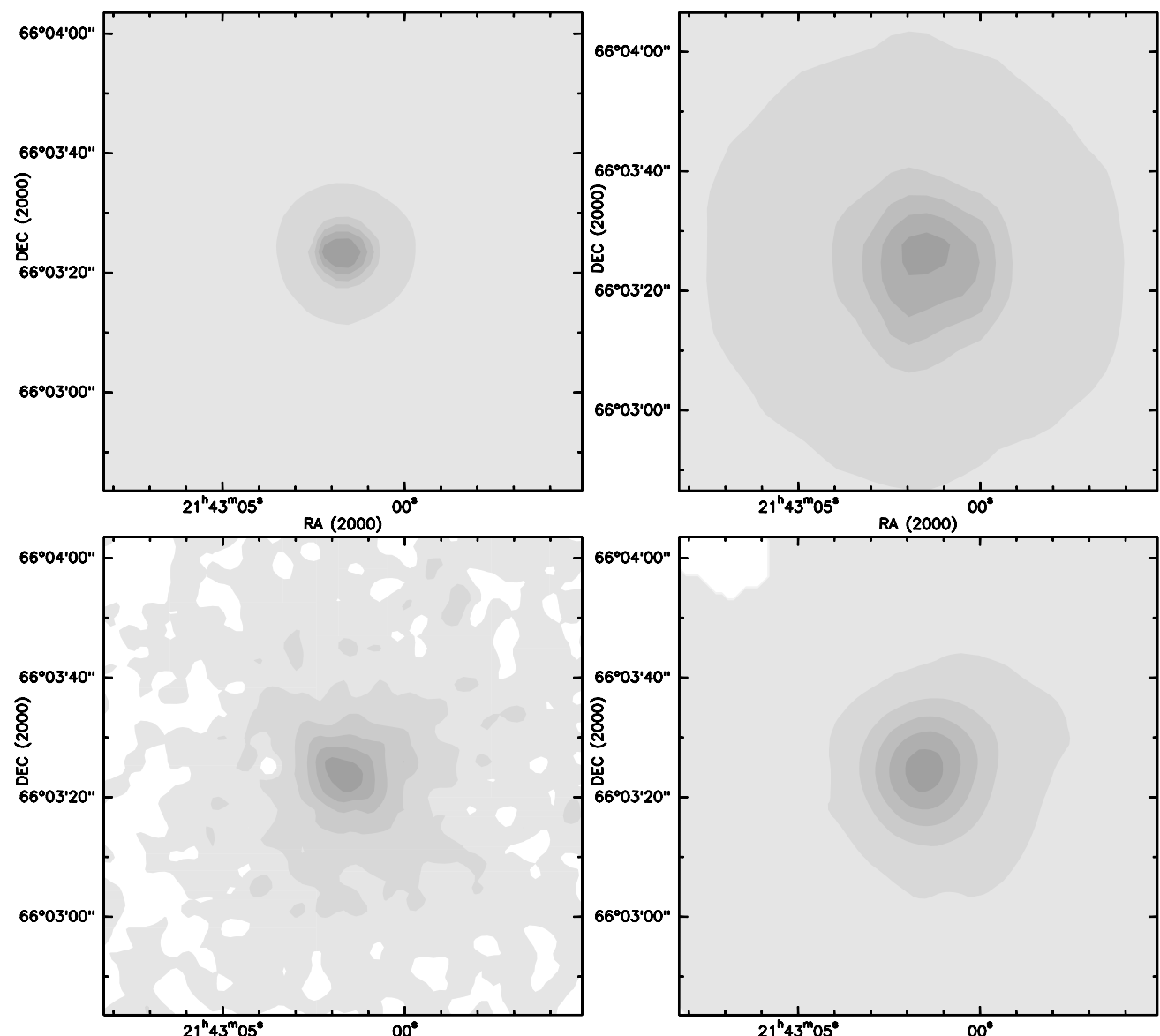

$66^{\circ} 04^{\prime} 00^{\prime \prime}$
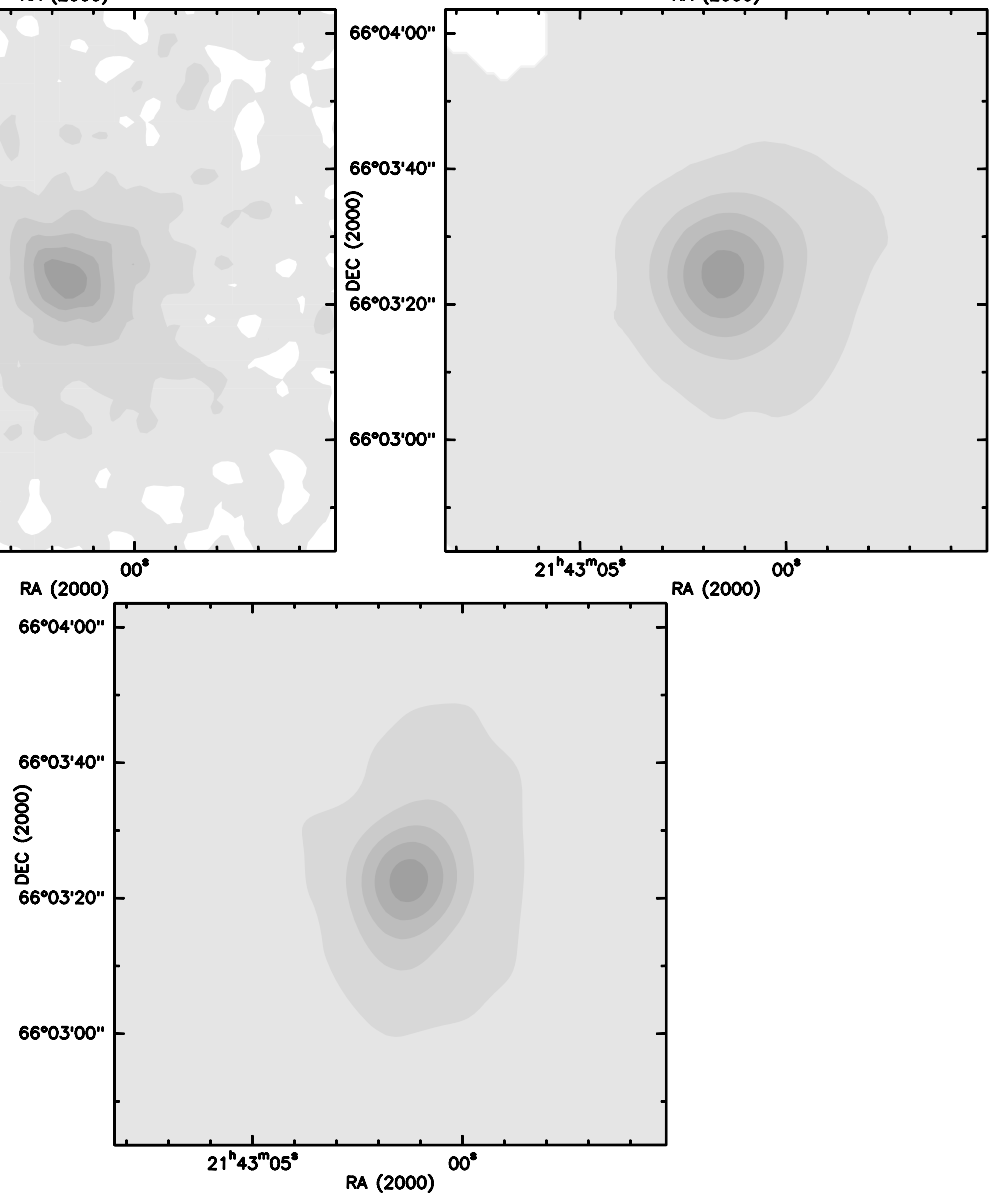

Fig. A.11. Continuum emission maps around NGC7129 FIRS 2 at $24 \mu \mathrm{m}$ (upper left panel), $70 \mu \mathrm{m}$ (upper right panel), $450 \mu \mathrm{m}$ (middle left panel), $850 \mu \mathrm{m}$ (middle right panel), and $1300 \mu \mathrm{m}$ (lower centred panel). The grey scale contours mark the continuum flux from $10 \%$ to $90 \%$ of the peak emission by steps of $20 \%$.

reported in Fig. A.13. The observed flux profiles and SED fluxes from $60 \mu \mathrm{m}$ to $850 \mu \mathrm{m}$ are well reproduced by the model. In minimising the $\chi_{\mathrm{SED}}^{2}$, we also varied the source luminosity from $400 L_{\odot}$ to $600 L_{\odot}$ and found the best-fit for a source luminosity equal to $500 L_{\odot}$. The observed flux at $24 \mu \mathrm{m}$ is underestimated by about two orders of magnitude. Considering the $\chi_{\text {red }}^{2}$ contour at $10 \%$ of the minimum $\chi_{\text {red }}^{2}$ to estimate the uncertainty, we obtained $r_{\text {out }}=(1.9 \pm 0.2) \times 10^{4} \mathrm{AU}$.

Finally, similarly to IC1396 N BIMA 2, the gas temperature profile obtained in NGC7129 FIRS 2 is slightly decoupled from the dust temperature in the inner part (see Fig. A.13).

\section{A.5. Serpens FIRS 1}

\section{A.5.1. Source background}

Since its recognition as a very active star forming region (Strom et al. 1974), the Serpens molecular cloud has been the target of many observational studies. Several studies have been dedicated to evaluate the distance of this cloud from the Sun, yielding a range of distances from $\sim 210$ to 440 pc. Following the review by Eiroa et al. (2008), which reports and compares the distance results from all the different studies, we adopt a distance of $\sim 230 \pm 20 \mathrm{pc}$. Serpens is a young protocluster whose members are in many different evolutionary stages. About 

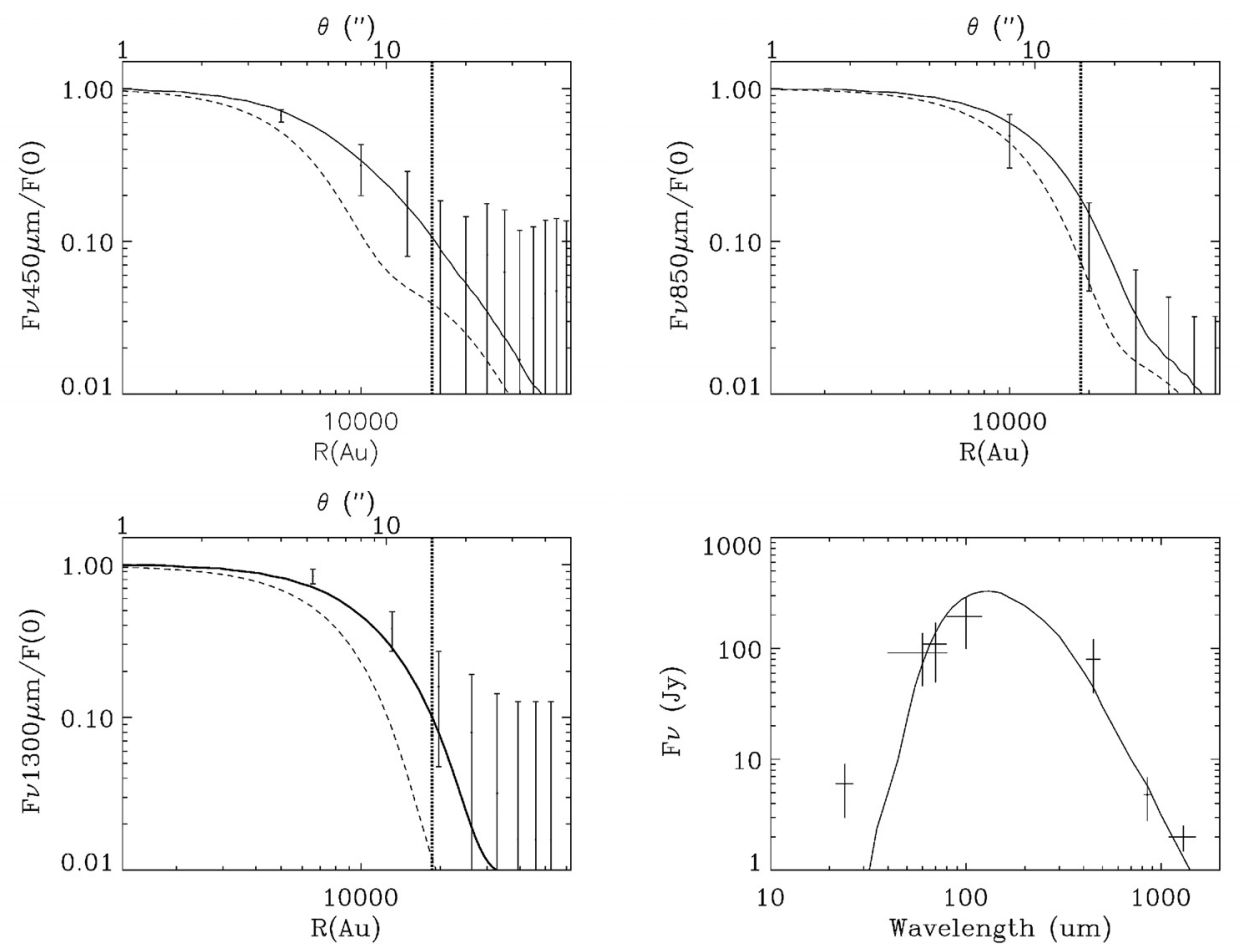

Fig. A.12. Observed brightness profiles at $450 \mu \mathrm{m}$ (upper left panel), $850 \mu \mathrm{m}$ (upper right panel), $1300 \mu \mathrm{m}$ (lower left panel), and the SED (lower right panel) toward NGC7129 FIRS 2. The solid lines report the best-fit model (Table 4). The dashed lines represent the beam pattern of the telescope at $450 \mu \mathrm{m}, 850 \mu \mathrm{m}, 1300 \mu \mathrm{m}$. The vertical dotted lines show the outer radius of the best-fit model.
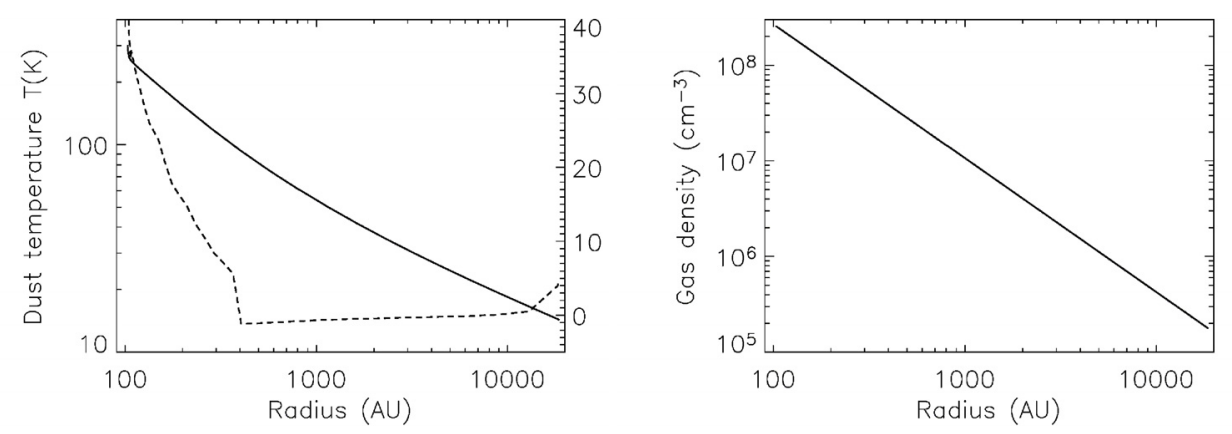

Fig. A.13. NGC7129 FIRS 2 dust temperature (left panel) and $\mathrm{H}_{2}$ density (right panel) profiles from the best-fit model. The dashed line represents the difference between the dust and gas temperature derived using $\mathrm{X}\left(\mathrm{H}_{2} \mathrm{O}\right)_{\text {in }}=1 \times 10^{-5}$ and $\mathrm{X}\left(\mathrm{H}_{2} \mathrm{O}\right)_{\text {out }}=1 \times 10^{-7}$ (scale on right axis).

100 embedded YSO, protostars, and prestellar clumps (Strom et al. 1976; Eiroa \& Casali 1992; Casali et al. 1993; Hurt \& Barsony 1996; Giovannetti et al. 1998; Testi \& Sargent 1998; Kaas 1999), as well as molecular outflows (White et al. 1995; Wolf-Chase et al. 1998; Williams \& Myers 2000) and $\mathrm{H}_{2}$ emission (e.g. Eiroa et al. 1997; Herbst et al. 1997; Hodapp 1999) have been detected in this region.

Serpens FIRS 1 is located near the centre of the Serpens main core and is the most luminous object embedded in the cloud. Several continuum studies classify it as Class 0 source with a bolometric luminosity estimated to range from $46 L_{\odot}$ to $84 L_{\odot}$ (Harvey et al. 1984; Casali et al. 1993; Hurt \& Barsony 1996; Larsson et al. 2000). The latest estimation of its luminosity suggests that Serpens FIRS 1 is on the low/intermediate mass protostar border. The envelope physical structure of Serpens FIRS 1 has been modelled by Hogerheijde et al. (1999) using millimetre interferometric observations of the continuum and molecular line observations. The Serpens FIRS 1 SED was first modelled by means of a spherical envelope $1 \mathrm{D}$ radiative transfer code by Larsson et al. (2000). Subsequently, Larsson et al. (2002) used a $2 \mathrm{D}$ radiative transfer code and a torus model to reproduce the SED and line observations from ISO. FIRS1 drives a molecular outflow, which is orientated at a position angle of 50 degrees, coincident with a triple radio source with symmetric lobes (Rodriguez et al. 1989).

\section{A.5.2. Analysis}

The continuum maps used for the Serpens FIRS 1 analysis are presented in Fig. A.14. The SCUBA maps were obtained in January 1998 as a part of the project $\mathrm{m} 97 \mathrm{bc} 30$. The flux profiles (see Fig. A.15) are derived excluding the dashed regions in Fig. A.14 to avoid the non-spherical extended emission. The Spitzer observations were obtained in October 2004 as part of the programme "From Molecular Cores to Planets, continued" (AOR: 174, PI: Neal Evans). The integrated fluxes used for the 
N. Crimier et al.: The dust physical structure of an Intermediate mass protostars sample
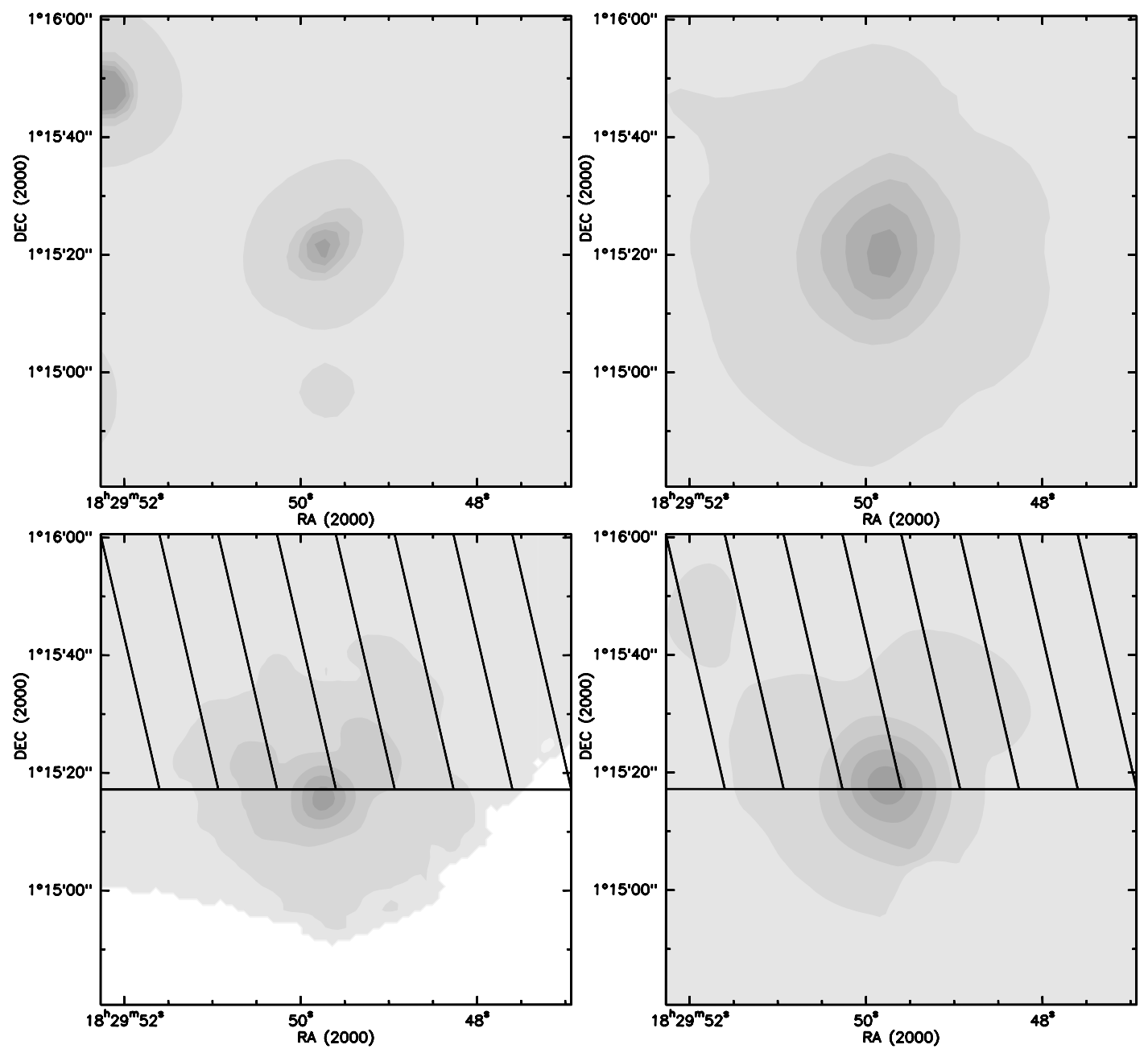

Fig. A.14. Continuum emission maps around Serpens FIRS 1 at $24 \mu \mathrm{m}$ (upper left panel), $70 \mu \mathrm{m}$ (upper right panel), $450 \mu \mathrm{m}$ (lower left panel), and $850 \mu \mathrm{m}$ (lower right panel). The grey scale contours mark the continuum flux from $10 \%$ to $90 \%$ of the peak emission by steps of $20 \%$. The dashed regions on the $450 \mu \mathrm{m}$ and $850 \mu \mathrm{m}$ show the regions excluded to derive the brightness profiles.

analysis are reported in Table 2 and in Fig. A.15. The integrated fluxes from $70 \mu \mathrm{m}$ to $850 \mu \mathrm{m}$ were obtained by integration over a $\sim 40^{\prime \prime}$. We also report on the SED the lower limits from Plateau de Bure (PdB) interferometric fluxes at $1.3 \mathrm{~mm}$ and $3 \mathrm{~mm}$ (Fuente et al. 2007).

\section{A.5.3. Best-fit}

Table 4 presents the set of parameters $\alpha, Y$, and $\tau_{100}$, which best reproduce the observations, and summarises some relevant physical quantities of the model. Figure A.15 shows the relevant derived brightness profiles and SED against the observations. The dust density and temperature profiles of the best-fit model are reported in Fig. A.16. The model well reproduces the observed flux profiles and SED fluxes from $70 \mu \mathrm{m}$ to $850 \mu \mathrm{m}$ and agrees well with the lower limits from Plateau de Bure interferometric fluxes at $1.3 \mathrm{~mm}$ and $3 \mathrm{~mm}$ (Fuente et al. 2007). In minimising the $\chi_{\text {SED }}^{2}$, we also varied the source luminosity from $20 L_{\odot}$ to $60 L_{\odot}$ and found the best-fit for a source luminosity equal to $33 L_{\odot}$. Note that the estimated value of the bolometric luminosity is much lower than the values derived in previous studies toward this source (Harvey et al. 1984; Casali et al. 1993; Hurt \& Barsony 1996; Larsson et al. 2000). This is due to the adopted smaller distance to the source. Considering the $\chi_{\text {red }}^{2}$ contour at
$10 \%$ of the minimum $\chi_{\text {red }}^{2}$ to estimate the uncertainty, we obtained $r_{\text {out }}=(5.9 \pm 0.3) \times 10^{3} \mathrm{AU}$.

Hogerheijde et al. (1999) studied the envelope physical structure of Serpens FIRS 1 using interferometric continuum observations at $\mathrm{OVRO}^{5}$ at $\lambda=3.4-1.4 \mathrm{~mm}$ and single-dish line observations from JCMT and $\mathrm{CSO}^{6}$. They adopted a bolometric luminosity of $77 L_{\odot}$ and fixed the outer radius of the envelope $R_{\text {out }}=8000$ AU for a distance of $400 \mathrm{pc}$. Hogerheijde et al. (1999) derived an envelope with density profile index $\alpha=2 \pm 0.5$ in good agreement with our results. By scaling their results to the same distance adopted here $(230 \mathrm{pc})$ their adopted luminosity, $\sim 25 L_{\odot}$, and the envelope radius $R_{\text {out }}, \sim 4600 \mathrm{AU}$, are relatively consistant with our results (Table 4 ): $L \sim 33 L_{\odot}, R_{\text {out }} \sim 5900 \mathrm{AU}$. Also their derived gas temperature profile slightly differs from ours: for example they obtained $T_{\text {gas }} \sim 40 \mathrm{~K}$ at $200 \mathrm{AU}$ while we obtained $T_{\text {gas }} \sim 65 \mathrm{~K}$. Larsson et al. (2000) studied the SED of Serpens FIRS 1, derived from the ISO LWS maps and photometric data between $\sim 1$ and $10^{5} \mu \mathrm{m}$ retrieved from the literature. These authors used i) a single temperature/optically thin approximation; and ii) a radiative transfer model to analyse the SED. In their models they adopted a distance of $310 \mathrm{pc}$, a source diameter of $7^{\prime \prime}$ and a power law density profile index, $\alpha=2$.

\footnotetext{
5 Owens Valley Radio Observatory.

${ }^{6}$ Caltech Submillimetre Observatory.
} 

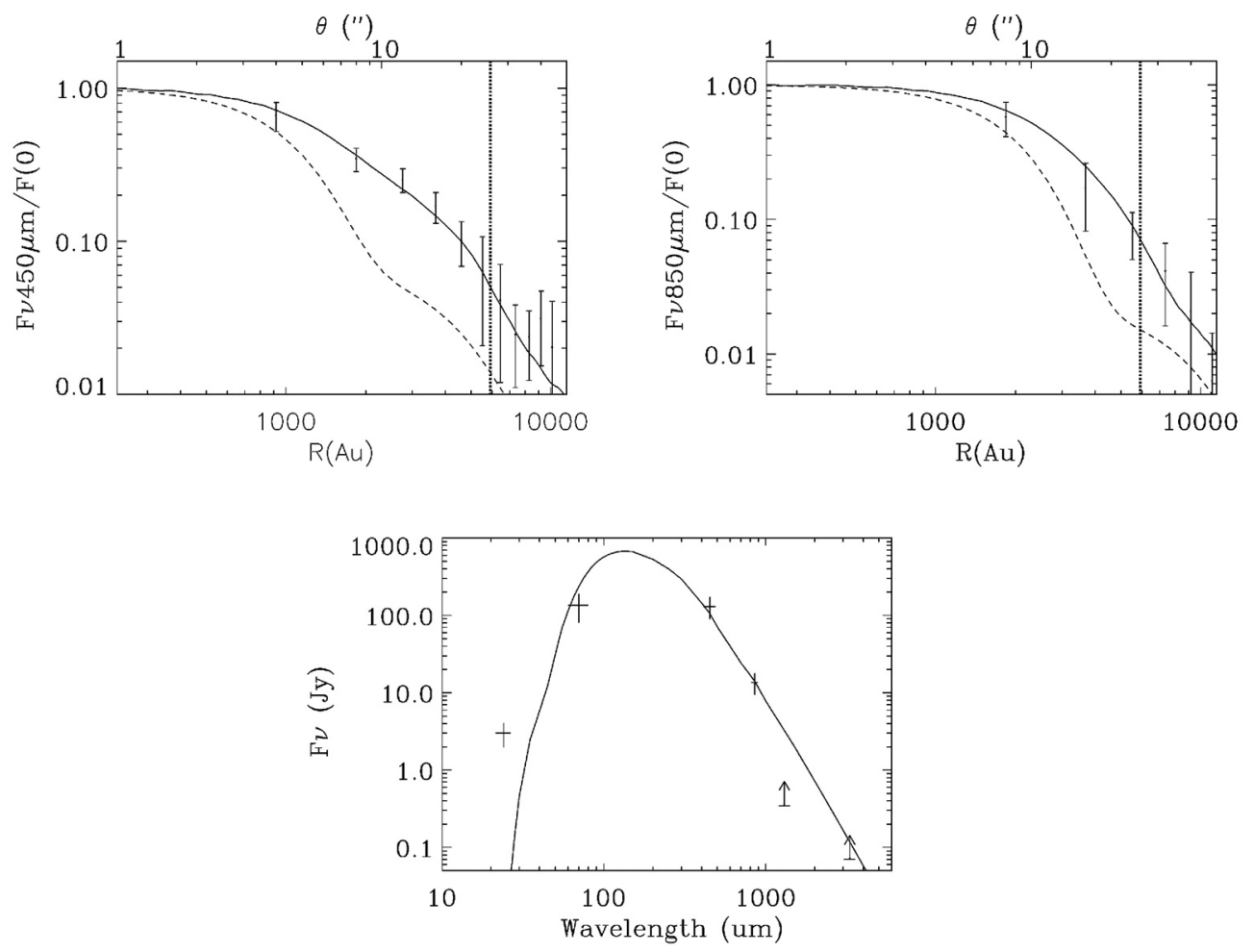

Fig. A.15. Observed brightness profiles at $450 \mu \mathrm{m}$ (upper left panel), $850 \mu \mathrm{m}$ (upper right panel) and the SED (lower centred panel) toward Serpens FIRS 1 . The solid lines report the best-fit model (Table 4). The dashed lines represent the beam pattern of the telescope at $450 \mu \mathrm{m}$ and $850 \mu \mathrm{m}$. The vertical dotted lines show the outer radius of the best-fit model. The lower limits from Plateau de Bure interferometric fluxes at $1.3 \mathrm{~mm}$ and $3 \mathrm{~mm}$ (Fuente et al. 2007) are also reported on the SED as lower limit.
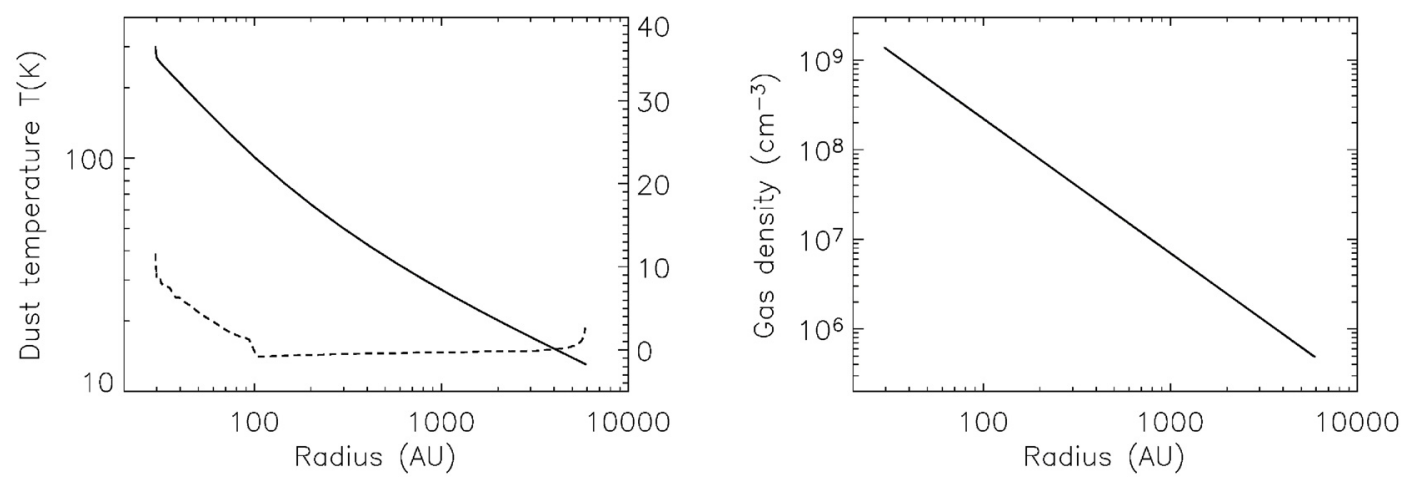

Fig. A.16. Serpens FIRS 1 dust temperature (left panel) and $\mathrm{H}_{2}$ density (right panel) profiles from the best-fit model. The dashed line represents the difference between the dust temperature and the gas temperature derived using $\mathrm{X}\left(\mathrm{H}_{2} \mathrm{O}\right)_{\text {in }}=1 \times 10^{-5}$ and $\mathrm{X}\left(\mathrm{H}_{2} \mathrm{O}\right)_{\text {out }}=1 \times 10^{-7}(\mathrm{scale}$ on right axis).

They succeeded in reproducing the SED between $40 \mu \mathrm{m}$ and $3 \mathrm{~mm}$. By scaling their results to the same distance adopted here (230 pc), they obtain a luminosity of $\sim 40 L_{\odot}$, against our estimate of $33 L_{\odot}$, and an envelope mass of $1.1-3.3 M_{\odot}$, against our estimate of $5 M_{\odot}$. However, as noticed by the authors themselves, their mass estimate is rather a lower limit, given the optically thin approximation they used.

Finally, the observed flux at $24 \mu \mathrm{m}$ is underestimated by about two orders of magnitude. This phenomenon is also observed in the Larsson et al. $(2002,2000)$ studies. We discuss the situation at $24 \mu \mathrm{m}$ in more detail in the the following section, Sect. 4.

\section{References}

Alonso-Albi et al. 2010, A\&A, accepted, 2

Andre, P., Ward-Thompson, D., \& Barsony, M. 1993, ApJ, 406, 122

Andre, P., Ward-Thompson, D., \& Barsony, M. 2000, Protostars and Planets IV, 59

Ayala, S., Noriega-Crespo, A., Garnavich, P. M., et al. 2000, AJ, 120, 909

Bechis, K. P., Harvey, P. M., Campbell, M. F., \& Hoffmann, W. F. 1978, ApJ, 226, 439

Beltrán, M. T., Girart, J. M., Estalella, R., Ho, P. T. P., \& Palau, A. 2002, ApJ, 573, 246

Beltrán, M. T., Girart, J. M., Estalella, R., \& Ho, P. T. P. 2004, A\&A, 426, 941

Beuther, H., Churchwell, E. B., McKee, C. F., \& Tan, J. C. 2007, in Protostars and Planets V, ed. B. Reipurth, D. Jewitt, \& K. Keil, 165 
N. Crimier et al.: The dust physical structure of an Intermediate mass protostars sample

Boogert, A. C. A., Pontoppidan, K. M., Lahuis, F., et al. 2004, ApJS, 154, 359 Bottinelli, S. 2006, Ph.D. Thesis, University of Hawai'i at Manoa Casali, M. M., Eiroa, C., \& Duncan, W. D. 1993, A\&A, 275, 195 Caux, E., Ceccarelli, C., Castets, A., et al. 1999, A\&A, 347, L1 Ceccarelli, C., Caselli, P., Herbst, E., Tielens, A. G. G. M., \& Caux, E. 2007, in Protostars and Planets V, ed. B. Reipurth, \& D. Jewitt, \& K. Keil, 47 Ceccarelli, C., Hollenbach, D. J., \& Tielens, A. G. G. M. 1996, ApJ, 471, 400 Ceccarelli, C., Maret, S., Tielens, A. G. G. M., Castets, A., \& Caux, E. 2003 , A\&A, 410, 587

Cesaroni, R., Felli, M., \& Walmsley, C. M. 1999, A\&AS, 136, 333

Chandler, C. J., Barsony, M., \& Moore, T. J. T. 1998, MNRAS, 299, 789

Chini, R., Ward-Thompson, D., Kirk, J. M., et al. 2001, A\&A, 369, 155

Codella, C., \& Bachiller, R. 1999, A\&A, 350, 659

Codella, C., Bachiller, R., Nisini, B., Saraceno, P., \& Testi, L. 2001, A\&A, 376, 271

Crimier, N., Ceccarelli, C., Lefloch, B., \& Faure, A. 2009, A\&A, 506, 1229

Crimier, N., Ceccarelli, C., Maret, S., et al. 2010, A\&A, accepted

de Gregorio-Monsalvo, I., Gómez, J. F., Suárez, O., et al. 2006, AJ, 132, 2584

Di Francesco, J., Evans, II, N. J., Harvey, P. M., et al. 1997, ApJ, 482, 433

Doty, S. D., \& Neufeld, D. A. 1997, ApJ, 489, 122

Draine, B. T., \& Li, A. 2001, ApJ, 551, 807

Eiroa, C., \& Casali, M. M. 1992, A\&A, 262, 468

Eiroa, C., Palacios, J., Eisloffel, J., Casali, M. M., \& Curiel, S. 1997, in HerbigHaro Flows and the Birth of Stars, ed. B. Reipurth \& C. Bertout, IAU Symp., 182,103

Eiroa, C., Palacios, J., \& Casali, M. M. 1998, A\&A, 335, 243

Eiroa, C., Djupvik, A. A., \& Casali, M. M. 2008, The Serpens Molecular Cloud, ed. B. Reipurth, 693

Eislöffel, J., Smith, M. D., Davis, C. J., \& Ray, T. P. 1996, AJ, 112, 2086

Evans, II, N. J., Rawlings, J. M. C., Shirley, Y. L., \& Mundy, L. G. 2001, ApJ, 557,193

Faure, A., \& Josselin, E. 2008, A\&A, 492, 257

Faure, A., Crimier, N., Ceccarelli, C., et al. 2007, A\&A, 472, 1029

Felli, M., Palagi, F., \& Tofani, G. 1992, A\&A, 255, 293

Flower, D. R., Pineau Des Forêts, G., \& Walmsley, C. M. 2006, A\&A, 449, 621

Frerking, M. A., Langer, W. D., \& Wilson, R. W. 1982, ApJ, 262, 590

Froebrich, D., Smith, M. D., Hodapp, K.-W., \& Eislöffel, J. 2003, MNRAS, 346, 163

Fuente, A., Neri, R., Martín-Pintado, J., et al. 2001, A\&A, 366, 873

Fuente, A., Neri, R., \& Caselli, P. 2005a, A\&A, 444, 481

Fuente, A., Rizzo, J. R., Caselli, P., Bachiller, R., \& Henkel, C. 2005b, A\&A, 433,535

Fuente, A., Ceccarelli, C., Neri, R., et al. 2007, A\&A, 468, L37

Fukui, Y., Iwata, T., Mizuno, A., Ogawa, H., \& Takaba, H. 1989, Nature, 342, 161

Giovannetti, P., Caux, E., Nadeau, D., \& Monin, J.-L. 1998, A\&A, 330, 990 Gomez, M., Hartmann, L., Kenyon, S. J., \& Hewett, R. 1993, AJ, 105, 1927

Gondhalekar, P. M., \& Wilson, R. 1975, A\&A, 38, 329

Greve, A., Kramer, C., \& Wild, W. 1998, A\&AS, 133, 271

Guesten, R., \& Marcaide, J. M. 1986, A\&A, 164, 342

Habing, H. J. 1968, BAIN, 19, 421

Hartigan, P., \& Lada, C. J. 1985, ApJS, 59, 383

Harvey, P. M., Wilking, B. A., \& Joy, M. 1984, ApJ, 278, 156

Hatchell, J., \& van der Tak, F. F. S. 2003, A\&A, 409, 589

Hennebelle, P., \& Fromang, S. 2008, A\&A, 477, 9

Herbst, T. M., Beckwith, S. V. W., \& Robberto, M. 1997, ApJ, 486, L59

Hillenbrand, L. A., \& Hartmann, L. W. 1998, ApJ, 492, 540

Hodapp, K. W. 1999, AJ, 118, 1338

Hogerheijde, M. R., van Dishoeck, E. F., Salverda, J. M., \& Blake, G. A. 1999, ApJ, 513, 350

Huard, T. L., Weintraub, D. A., \& Sandell, G. 2000, A\&A, 362, 635

Hurt, R. L., \& Barsony, M. 1996, ApJ, 460, L45

Ivezic, Z., \& Elitzur, M. 1997, MNRAS, 287, 799

Jørgensen, J. K., Schöier, F. L., \& van Dishoeck, E. F. 2002, A\&A, 389, 908

Jørgensen, J. K., Lahuis, F., Schöier, F. L., et al. 2005, ApJ, 631, L77

Jørgensen, J. K., Bourke, T. L., Myers, P. C., et al. 2007, ApJ, 659, 479

Kaas, A. A. 1999 , AJ, 118, 558

Krumholz, M. R., McKee, C. F., \& Klein, R. I. 2005, ApJ, 618, L33

Ladd, E. F., \& Hodapp, K.-W. 1997, ApJ, 474, 749

Larsson, B., Liseau, R., Men'shchikov, A. B., et al. 2000, A\&A, 363, 253

Larsson, B., Liseau, R., \& Men'shchikov, A. B. 2002, A\&A, 386, 1055

Launhardt, R., \& Henning, T. 1997, A\&A, 326, 329
Launhardt, R., Evans, II, N. J., Wang, Y., et al. 1998, ApJS, 119, 59

Lefloch, B., Eisloeffel, J., \& Lazareff, B. 1996, A\&A, 313, L17

Li, Z., \& Shu, F. H. 1996, ApJ, 472, 211

Lizano, S., \& Shu, F. H. 1989, ApJ, 342, 834

Mannings, V., \& Sargent, A. I. 1997, ApJ, 490, 792

Mannings, V., \& Sargent, A. I. 2000, ApJ, 529, 391

Matthews, H. I. 1979, A\&A, 75, 345

McKee, C. F., \& Tan, J. C. 2003, ApJ, 585, 850

McLaughlin, D. E., \& Pudritz, R. E. 1996, ApJ, 469, 194

McLaughlin, D. E., \& Pudritz, R. E. 1997, ApJ, 476, 750

Miranda, L. F., Eiroa, C., \& Gomez de Castro, A. I. 1993, A\&A, 271, 564

Moro-Martín, A., Noriega-Crespo, A., Molinari, S., et al. 2001, ApJ, 555, 146

Motte, F., \& André, P. 2001, A\&A, 365, 440

Mueller, K. E., Shirley, Y. L., Evans, II, N. J., \& Jacobson, H. R. 2002, ApJS, 143,469

Neri, R., Fuente, A., Ceccarelli, C., et al. 2007, A\&A, 468, L33

Nisini, B., Massi, F., Vitali, F., et al. 2001, A\&A, 376, 553

Noriega-Crespo, A., \& Garnavich, P. M. 2001, AJ, 122, 3317

Noriega-Crespo, A., Moro-Martin, A., Carey, S., et al. 2005, in ESA SP 577, ed. A. Wilson, 453

Osorio, M., Lizano, S., \& D’Alessio, P. 1999, ApJ, 525, 808

Osterbrock, D. E. 1957, ApJ, 125, 622

Ossenkopf, V., \& Henning, T. 1994, A\&A, 291, 943

Palla, F., Cesaroni, R., Brand, J., et al. 1993, A\&A, 280, 599

Parise, B., Ceccarelli, C., \& Maret, S. 2005, A\&A, 441, 171

Patel, N. A., Greenhill, L. J., Herrnstein, J., et al. 2000, ApJ, 538, 268

Rodriguez, L. F., Moran, J. M., Ho, P. T. P., \& Gottlieb, E. W. 1980, ApJ, 235, 845

Rodriguez, L. F., Curiel, S., Moran, J. M., et al. 1989, ApJ, 346, L85

Sandell, G., \& Weintraub, D. A. 2001, ApJS, 134, 115

Saraceno, P., Ceccarelli, C., Clegg, P., et al. 1996, A\&A, 315, L293

Schöier, F. L., Jørgensen, J. K., van Dishoeck, E. F., \& Blake, G. A. 2002, A\&A, 390,1001

Schreyer, K., Henning, T., van der Tak, F. F. S., Boonman, A. M. S., \& van Dishoeck, E. F. 2002, A\&A, 394, 561

Sellgren, K., Werner, M. W., \& Dinerstein, H. L. 1983, ApJ, 271, L13

Serabyn, E., Guesten, R., \& Mundy, L. 1993, ApJ, 404, 247

Shevchenko, V. S., \& Yakubov, S. D. 1989, SvA, 33, 370

Shirley, Y. L., Evans, II, N. J., \& Rawlings, J. M. C. 2002, ApJ, 575, 337

Shu, F. H. 1977, ApJ, 214, 488

Shu, F. H., \& Adams, F. C. 1987, in Circumstellar Matter, ed. I. Appenzeller, \& C. Jordan, IAU Symp., 122, 7

Smith, M. D., Froebrich, D., \& Eislöffel, J. 2003, ApJ, 592, 245

Stahler, S. W., Palla, F., \& Salpeter, E. E. 1986, ApJ, 302, 590

Strom, S. E., Grasdalen, G. L., \& Strom, K. M. 1974, ApJ, 191, 111

Strom, S. E., Vrba, F. J., \& Strom, K. M. 1976, AJ, 81, 638

Sugitani, K., Fukui, Y., Mizuni, A., \& Ohashi, N. 1989, ApJ, 342, L87

Sugitani, K., Matsuo, H., Nakano, M., Tamura, M., \& Ogura, K. 2000, AJ, 119, 323

Testi, L., \& Sargent, A. I. 1998, ApJ, 508, L91

Testi, L., Palla, F., \& Natta, A. 1999, A\&A, 342, 515

Tofani, G., Felli, M., Taylor, G. B., \& Hunter, T. R. 1995, A\&AS, 112, 299

Troscompt, N., Faure, A., Wiesenfeld, L., Ceccarelli, C., \& Valiron, P. 2009, A\&A, 493, 687

van der Tak, F. F. S., van Dishoeck, E. F., Evans, II, N. J., Bakker, E. J., \& Blake, G. A. 1999, ApJ, 522, 991

Van der Tak, F. F. S., van Dishoeck, E. F., Evans, II, N. J., \& Blake, G. A. 2000, ApJ, 537, 283

Vastel, C., Caux, E., Ceccarelli, C., et al. 2000, A\&A, 357, 994

Visser, A. E., Richer, J. S., \& Chandler, C. J. 2002, AJ, 124, 2756

Wang, Y., Evans, II, N. J., Zhou, S., \& Clemens, D. P. 1995, ApJ, 454, 217

Weikard, H., Wouterloot, J. G. A., Castets, A., Winnewisser, G., \& Sugitani, K. 1996, A\&A, 309, 581

White, G. J., Casali, M. M., \& Eiroa, C. 1995, A\&A, 298, 594

Wilking, B., Mundy, L., McMullin, J., Hezel, T., \& Keene, J. 1993, AJ, 106, 250

Williams, J. P., \& Myers, P. C. 2000, ApJ, 537, 891

Williams, S. J., Fuller, G. A., \& Sridharan, T. K. 2005, A\&A, 434, 257

Wolf-Chase, G. A., Barsony, M., Wootten, H. A., et al. 1998, ApJ, 501, L193

Young, C. H., Shirley, Y. L., Evans, II, N. J., \& Rawlings, J. M. C. 2003, ApJS, 145,111

Yun, J. L., \& Clemens, D. P. 1994, AJ, 108, 612

Yun, J. L., \& Clemens, D. P. 1995, AJ, 109, 742 